\title{
An experimental study of geometrical effects on the drag and flow field of two bluff bodies separated by a gap
}

\author{
By KEITH KOENIG † AND ANATOL ROSHKO \\ California Institute of Technology, Pasadena, California 91125
}

(Received 11 July 1984)

This paper describes an experimental investigation of the shielding effects of various disks placed coaxially upstream of an axisymmetric, flat-faced cylinder. Remarkable decrease of the drag of such a system was observed for certain combinations of the basic geometric parameters, namely the diameter and gap ratios. For such optimum shielding the stream surface which separates from the disk reattaches smoothly onto the front edge of the cylinder, in what is close to a 'free-streamline' flow; alternatively, the flow may be viewed as a cavity flow. For the optimum as well as other geometries, flow pictures, pressure distributions and some LDV measurements were also obtained. From these, several flow regimes depending on the gap/diameter parameters were identified. Variations on the axisymmetric disk-cylinder configuration included a hemispherical frontbody, rounding of the front edge of the cylinder and a change from circular to square cross-section.

\section{Introduction}

Problems involving the flow over two bluff bodies which interfere with each other have received relatively little attention from researchers, perhaps because the flow over an isolated bluff body is already difficult enough. A simple example of such an interfering flow is that in which one body is far downstream in the wake of another one. In this case the drag of the downstream body is reduced owing to the reduced dynamic pressure in the wake in which it is immersed, while the drag of the first body is unaffected. This may be called a weak interaction. When the downstream body is brought close to the base of the first one, the drag of the former may be significantly reduced. In addition, the drag of the first body may be strongly affected; this would be a strong interaction. There appear to be no good guidelines for estimating such effects, yet there are many practical problems in which one bluff body is shielded by another one.

While not attempting a complete review, we note that possibly the first investigation of this kind was made by Eiffel (1910), who studied the effect of spacing on the drag of two disks arranged coaxially in a stream. This configuration has recently been investigated again, in more detail, by Morel \& Bohn (1980). In connection with an investigation to reduce the drag of a tractor-trailer rig by means of a shield on the roof of the tractor, Saunders (1966) studied the overall drag on a prism of circular cross-section shielded by a disk placed coaxially upstream. A special case (figure 1) of Saunder's configuration is the main subject of the present investigation. The inverse case, a disk placed in the wake of a coaxial cylinder, was investigated by Mair

$\dagger$ Now at Aerospace Engineering, Mississippi State, Mississippi 39762. 


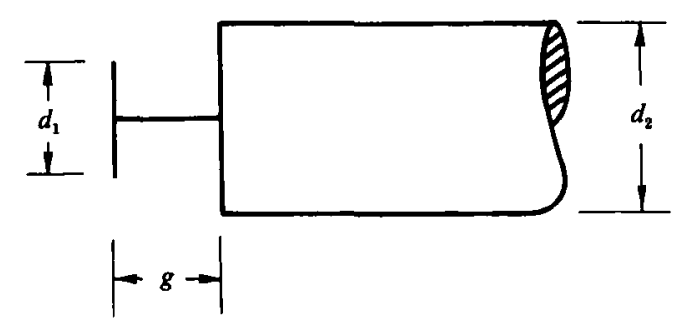

Figure 1. The configuration studied: a disk and a coaxial, semi-infinite cylinder of circular cross-section, separated by a gap.

(1965) and further by Little \& Whipkey (1979). The above examples are all for axisymmetric flows. The case of flow interference between two parallel circular cylinders with axes normal to the approaching flow has been reviewed by Zdravkovich (1977).

Apart from possible practical applications, studies of flow over interfering body configurations can be of interest in their own right for the insights they may give into separated flow phenomena.

The configuration which was investigated in the present work (figures 1 and 3 ) consisted of a circular cylinder with axis parallel to a wind-tunnel free stream and a disk placed coaxially upstream of the flat face of the cylinder. The diameters of the disk and cylinder are $d_{1}$ and $d_{2}$, respectively, and $g$ is the gap between them. Some observations and measurements were also made for other upstream-body shapes. In the absence of the upstream body, the circular cylinder in figure 1 has a pressure force on its upstream face which makes a contribution of about 0.75 to the drag coefficient (based on the cross-sectional area). This drag is connected with loss of suction near the edge of the front face, a consequence of separation from that edge. In potential flow in an infinite stream such a cylinder, assuming it to be an infinite half body, would have zero drag, the high pressure near the centre of the face being balanced by the suction near the edge. This is true in fact for any semi-infinite body, whatever the nose shape (Prandtl \& Tietjens 1934; Morel 1978). Thus, such a configuration is attractive for attempting to elucidate the effect of an upstream interfering body because the flow in the region of interest is not complicated by coupling to a base flow downstream.

Even with this simplification the possible flow patterns can be expected to be quite complex, with separation streamlines usually springing from the edges of both the cylinder and the disk. As a first attempt to classify the possibilities, we prepared figure 2 in which different conceivable flow patterns formed by the separating/reattaching streamlines from the frontbody are depicted qualitatively. The patterns were drawn by simply superimposing on a disk-wake profile the rectangular rearbody profile, varying the diameter and gap. For the disk-wake profile, the experimental result of Carmody (1964) has been used. In the real flow, of course, the disk wake will be strongly altered when the gap between the bodies is not large and this classification could at best be only a rough guide to the actual flow patterns that occur. Nevertheless the classification of flow patterns shown in figure 2 provides an interesting and useful reference for examining the flows that were observed in the experiments to be described. Of the nine different flow patterns defined in figure 2, four correspond to the four flow regimes denoted by $A, B, C$, and $\mathrm{D}$ in the $(g, d)$-plane; four others to the boundaries $\mathrm{AB}, \mathrm{CB}, \mathrm{CD}$ and $\mathrm{BD}$ between those 
(a)
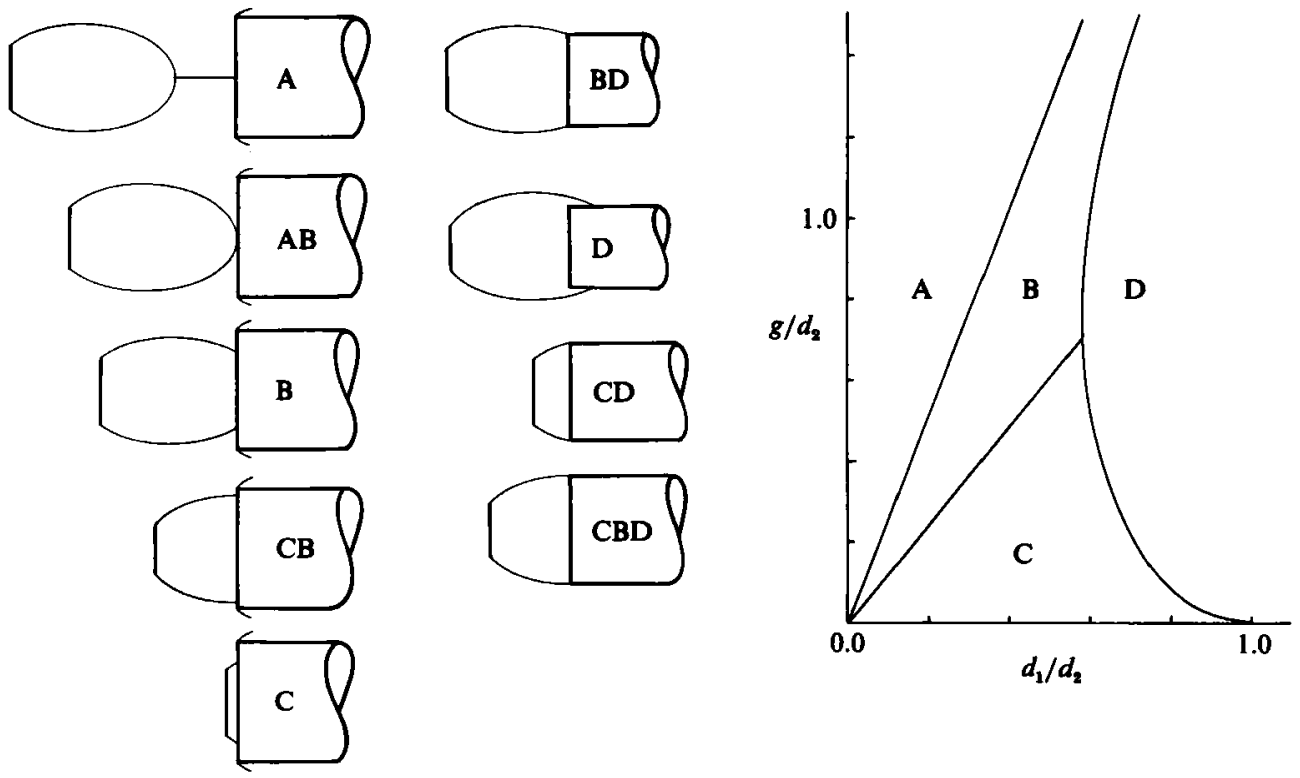

Figure 2. (a) Patterns obtained from intersections of a disk-wake profile and a semi-infinite cylinder. (b) Corresponding regimes in the $\left(g, d_{1}\right)$-plane.

regimes; and the ninth (CBD) to the common point between three of them. This latter occurs for a particular pair of values of the diameter $d_{1} / d_{2}$ and gap ratio $g / d_{2}$. In this particular configuration the disk wake joins smoothly (tangentially) onto the cylinder. As will be seen, such a flow pattern actually occurs (though not at exactly those values) and corresponds to very low values of the drag.

\section{Experimental details}

The experiments were carried out using two models, one designed for force and pressure measurements in a wind tunnel and the other for flow visualization and laser-Doppler velocimetry in a water tunnel.

The configuration shown in figure 3 was built with $d_{2}=8 \mathrm{in}$. $(20.3 \mathrm{~cm})$ for installation in the GALCIT $\dagger$ Merrill Wind Tunnel which has a test section 32 in. $\times 46$ in. $(81 \mathrm{~cm} \times 117 \mathrm{~cm})$ in cross-section and $104 \mathrm{in} .(264 \mathrm{~cm})$ long; its turbulence level is about $\frac{1}{2} \%$. The model had two major parts - a metric forward portion which was connected to a force balance and a non-metric rear portion rigidly mounted to the tunnel walls. The metric portion was composed of a frontbody, sting and a short section of the rearbody. For the majority of the measurements the rearbody was a circular cylinder of 8 in. $(20.3 \mathrm{~cm})$ diameter and $32 \mathrm{in} .(81.2 \mathrm{~cm})$ length. The length of the rearbody, 4 diameters, was enough to ensure adequate approximation to a semi-infinite body. Measurements of the distribution of pressure on the side of a long, flat-faced cylinder (e.g. Ota 1975) show that the pressure returns to free-stream values within 2 diameters downstream. The frontbodies were mainly flat disks, $\frac{1}{8}$ in. 


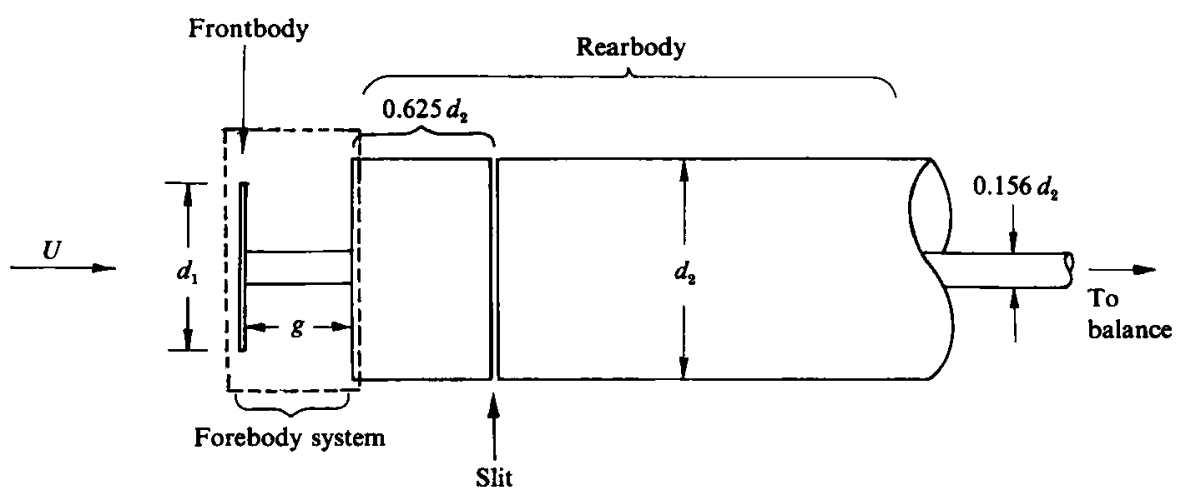

Frgure 3. Schematic diagram of the experimental model.

$(0.32 \mathrm{~cm})$ thick, varying in diameter from 2 to $8 \mathrm{in} .(5-20.3 \mathrm{~cm})$, mounted coaxially in front of the rearbody on a $1 \frac{1}{4} \mathrm{in} .(3.1 \mathrm{~cm})$ diameter sting. A hemisphere of $8 \mathrm{in}$. $(20.3 \mathrm{~cm})$ diameter was also used. The rearbody face was replaceable, as were the frontbodies. Some measurements were also made on a rearbody of square cross-section with square-plate frontbodies.

The central sting supporting the metric portion of the model was mounted on a force balance which provided direct analog readout of the three aerodynamic forces and the three moments acting on the supported model. Pressure distributions on the face of the rearbody and in the slot between the metric and dummy portions of the model were measured, using $\frac{1}{32}$ in. $(0.08 \mathrm{~cm})$ pressure orifices and a $0-100 \mathrm{mmHg}$ Barocel pressure transducer. The transducer output was measured on a HewlettPackard Timer-Counter DVM with a variable integration time up to $10 \mathrm{~s}$. To determine the symmetry of the flow, pressure taps on the rearbody face were arranged along several radii and side and lift forces were monitored. It was possible with these observations to detect misalignment of the model with the freestream of less than $0.5^{\circ}$ (Koenig 1978). All results presented here are for flow/model inclinations of less than $0.5^{\circ}$. The force and pressure measurements were made at speeds from 25 to $190 \mathrm{ft} / \mathrm{s}(7.6-57.9 \mathrm{~m} / \mathrm{s})$; the corresponding values of the Reynolds number $R e$ based on $d_{2}$ were $1 \times 10^{5}-8 \times 10^{5}$. Except for a very few cases, to be discussed, no significant dependence of the drag coefficient or the pressure coefficient distributions on Reynolds number for the range $R e=1 \times 10^{5}-8 \times 10^{5}$ was observed in these experiments. All geometries were investigated over this Reynolds-number range; checks to verify observations and determine repeatability were concentrated, however, at $R e=5 \times 10^{5}$. The results to follow are, therefore, at $R e=5 \times 10^{5}$ unless otherwise noted. Repeatability of the measured forebody-system drag coefficient, $C_{D_{m}}$, in these experiments was estimated to be \pm 0.004 .

A second model, with $d_{2}=4 \mathrm{in} .(10.2 \mathrm{~cm})$, was built for installation in the GALCIT Free Surface Water Tunnel (Ward 1976). This model was used for flow-visualization experiments and laser-Doppler velocity measurements. For the flow visualization, diluted food colouring was injected into the flow from the face of the frontbody, the face of the rearbody, or from an upstream probe. These experiments were made at a water speed of $3.5 \mathrm{ft} / \mathrm{s}(1.1 \mathrm{~m} / \mathrm{s})$ giving a Reynolds number of $1 \times 10^{5}$.

Our intent in these experiments was to investigate the behaviour of the forward portion of a frontbody-rearbody combination. Specifically, the system of interest is the entire frontbody and the face of the rearbody (see figure 3), which we define as 


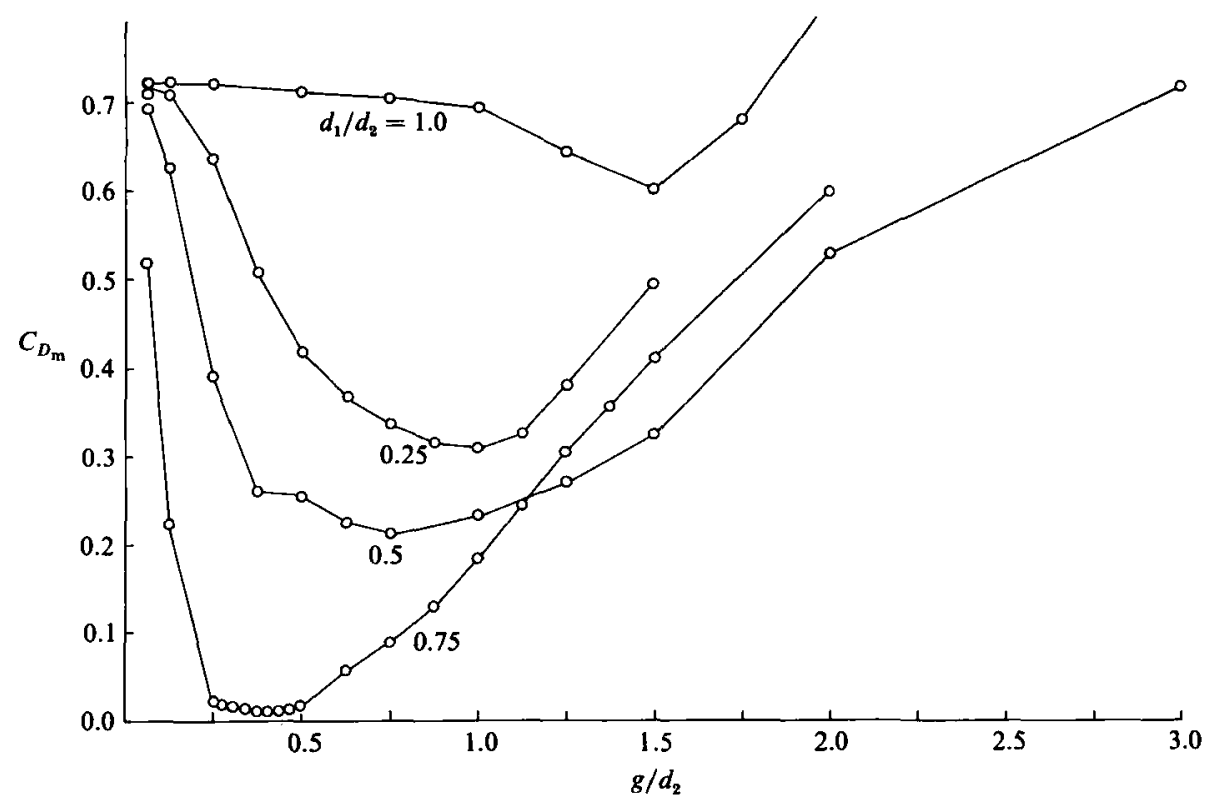

Frgure 4. Typical measured results for the axisymmetric forebody-system drag coefficient: reference area $=\frac{1}{4} \pi d_{2}^{2}$; Reynolds number, $R e=U_{\infty} d_{2} / \nu=500000$.

the forebody system. For practical reasons it was necessary to provide a connection (sting) between the frontbody and rearbody and to include a short segment of the rearbody downstream of its face as a part of the metric model (figure 3). Consequently the measured drag includes contributions from the sting and the sides and base of the short rearbody segment. The pressure force on the base of the short rearbody segment was subtracted from the drag balance measurement, using the pressure measured in the gap between that and the static part of the rearbody. The measured forebody system drag coefficient is then defined as

$$
C_{D \mathrm{~m}}=\frac{\text { force from balance }-\int_{(\text {base })}\left(p-p_{\infty}\right) \mathrm{d} A}{\frac{1}{2} \rho U_{\infty}^{2} \frac{1}{4} \pi d_{2}^{2}} .
$$

In what follows, the drag measurements as determined from this definition are presented without correction for blockage or for extraneous skin friction on the short metric segment of the rearbody. This allows an unprejudiced record of the measurements and is sufficient for a comparative study of the effects of various parameters. Where it is important to estimate the free-stream values of $C_{D}$ more accurately, especially the very low values, corrections for blockage and extraneous skin friction are applied, as described in the Appendix, and the resulting, corrected value is denoted by $C_{D_{\mathbf{c}}}$.

\section{Forebody drag coefficients for circular cross-section}

The value of $C_{D_{\mathrm{m}}}$ of the flat-faced cylinder when free from a frontbody was found to be 0.72. With the blockage correction (Appendix) the corrected value is found to be $C_{D_{\mathrm{c}}}=0.75$. Values ranging from $0.7-0.8$, obtained by various methods, have been reported by Polhamus (1957), Stanbrook (1964), Hoerner (1965) and Norris \& 

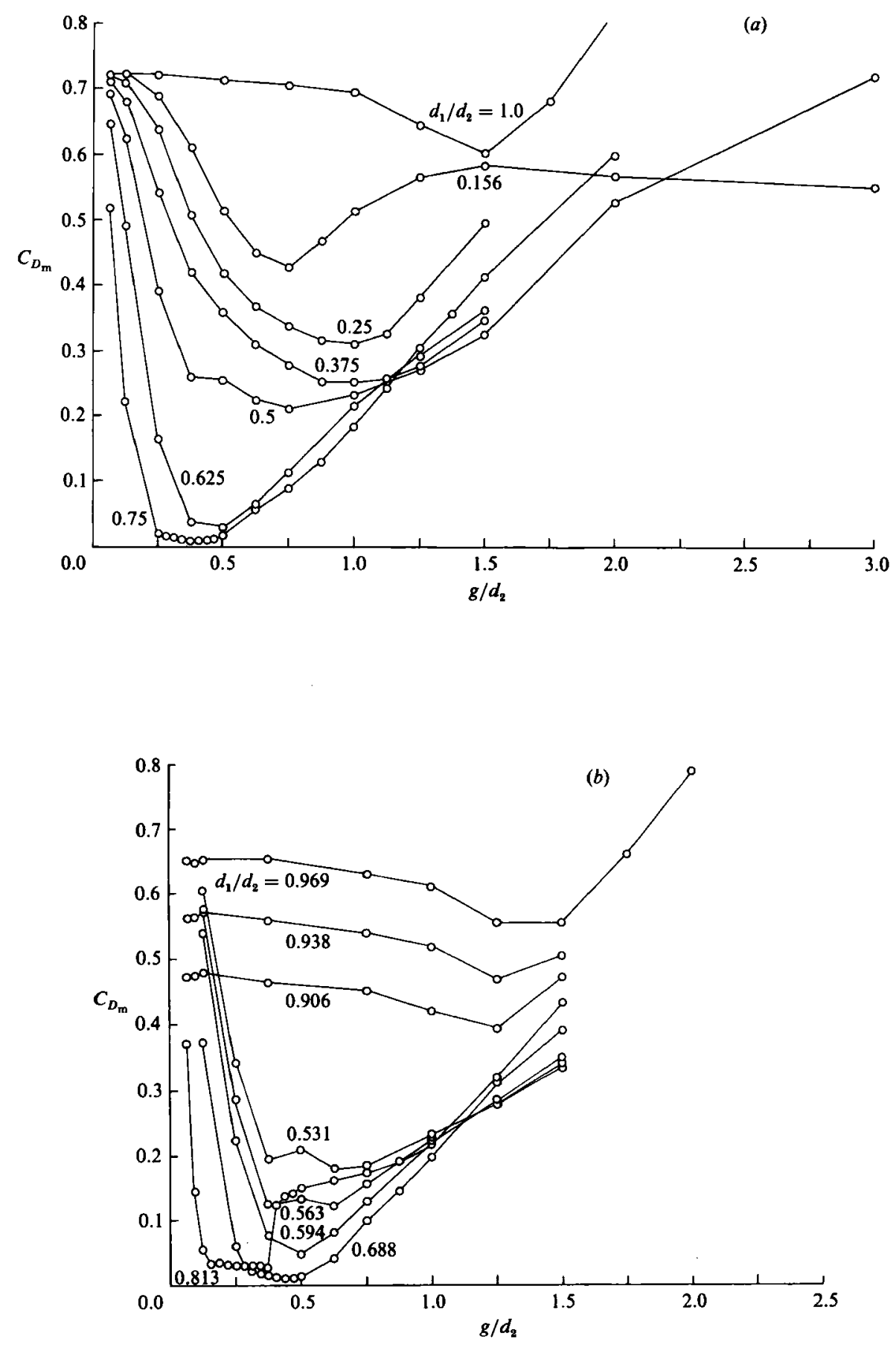

Figure $5(a, b)$. For description see opposite. 


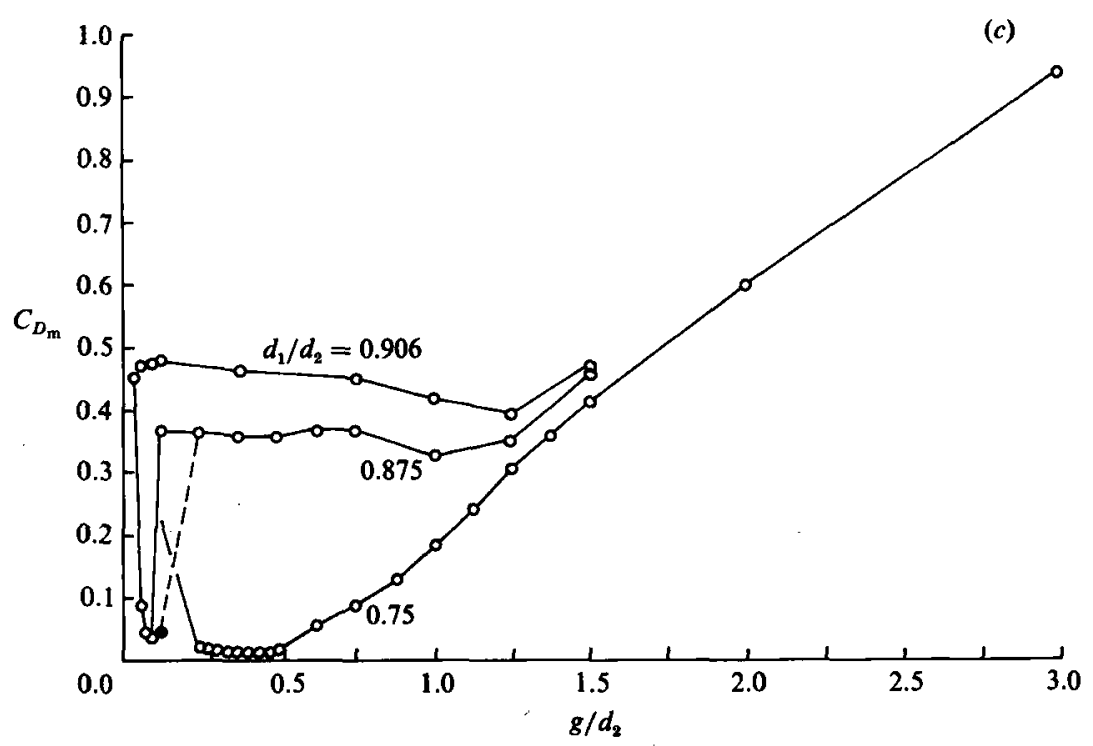

Figure 5. Measured axisymmetric-forebody drag coefficient. (a), (b) $R e=500000 ;$ (c) $\bigcirc, R e=500000 ; \bigcirc, 650000$.

McGhee (1966). Besides measurement uncertainties, some of this variation may be due to differences in freestream turbulence level and scale (Bearman 1971).

Placing flat disks of various diameters at various gap distances ahead of the cylinder face produces large variations in the forebody-system drag coefficient, as shown for several disks in figure 4. These are all for Reynolds number $R e=5 \times 10^{5}$, based on $d_{2}$. For $g / d_{2} \rightarrow 0$, values of $C_{D_{m}}$ for all values of $d_{1} / d_{2}<1$ tend to 0.72 , as expected. As the gap ratio increases, there is a rapid decrease in $C_{D_{\mathrm{m}}}$ as the shielding effect of the frontbody develops. For each disk, a minimum value of $C_{D_{\mathrm{m}}}$ is attained; with further increase of $g / d_{2}$ the forebody system drag rises, presumably approaching eventually the sum of the free-field values of the two bodies for $g / d_{2} \rightarrow \infty$. A minimum value of $C_{D_{m}}$ occurs for each value of $d_{1} / d_{2}$. What is remarkable is that for some configurations the drag of the forebody system is extremely low. In particular, for $d_{1} / d_{2}=0.75$ and $g / d_{2}=0.375$ the measured value of $C_{D_{m}}$ was 0.01 . (After correction for blockage and extraneous skin friction, $C_{D_{c}}$ is estimated to be 0.03.) Although the uncertainty of this low value may be as much as $50 \%$, it is clear that the drag of this particular disk-gap-rearbody configuration is comparable to that of a solid forebody with no separation.

The minimum value, for each value of $d_{1} / d_{2}$, will be denoted by $C_{D}^{*}$ and the corresponding gap ratio by $g^{*} / d_{2}$. (Because the minima are relatively broad, it is sometimes difficult to define the value of $g^{*}$ precisely.) These values and the associated configurations will be called 'optimum'.

The complete set of results, for all the flat disks used, is presented in figure 5 . The general observations on figure 4 may be seen to apply to a wide range of forebody parameters. Some exceptions to the generally smooth variation with changing $g / d_{2}$ are observed for values of $d_{1} / d_{2}=0.531,0.563$, and 0.875 . The first two of these show closely spaced minima in $C_{D}$, the one at larger $g / d_{2}$ being lower. These measurements were repeatable, the non-smoothness apparently not due to experimental error or 

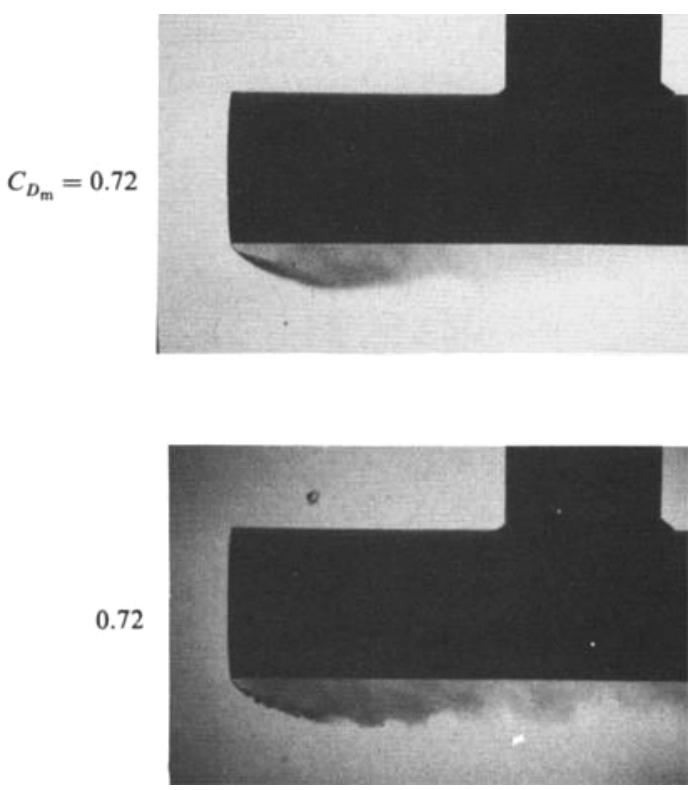

Figure 6. Visualization of rearbody alone.

seatter. Flow visualization indicated that the flow in these configurations was rather unsteady.

The curve for $d_{1} / d_{2}=0.875$ has a sharp minimum at $g / d_{2}=0.094$ and another shallower one at $g / d_{2}=1.0$, both quite repeatable. At $g / d_{2}=0.094$ the flow was very steady and stable, resisting even severe attempts to perturb it. The result was also insensitive to Reynolds number, over the range $1-8 \times 10^{6}$, and to roughness (no. 120 sandpaper) on the face of the disk. On the other hand, for $g / d_{2}=0.125$, the flow condition was sensitive to $R e$ and roughness, jumping from the higher- to the lower-drag regime with increasing $R e$ and vice versa with decreasing $R e$, but with hysteresis. The solid point in figure $5(c)$ corresponds to the low-drag regime attained for $R e>6.7 \times 10^{5}$, with roughness. It should also be noted that for the curve labelled $d_{1} / d_{2}=0.156$ the 'disk' was the face of the sting, the diameter of the latter being $0.156 d_{2}$.

\section{Flow patterns and pressure distributions}

Using diluted food colouring injected through the upstream face of the model in the water tunnel the pictures in figures 6 and 7 were obtained. It should be noted that on some of the pictures the dye flow is biased to one side, and the flow pattern is exhibited more weakly or not at all on the other side.

The cylinder without frontbody is illustrated in figure 6, where the large region of separation, originating at the shoulder, may be seen. It corresponds well with observations in the wind tunnel, where tuft and surface probe measurements indicated a flow-reversal region extending $1 \frac{1}{2}$ diameters downstream of the shoulder.

Flow patterns with frontbody in place are illustrated in figure 7 for several different disks placed at various distances upstream of the cylinder. In $\$ 9$ these flow patterns are compared with those postulated in figure 2. Photographic exposure times are 
(a)

$$
\begin{array}{r}
g / d_{2}=1.625 \\
C_{D_{\mathrm{m}}}=(0.55)
\end{array}
$$

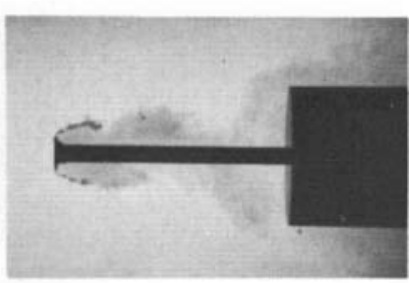

$* 1.0$

$(0.31)$

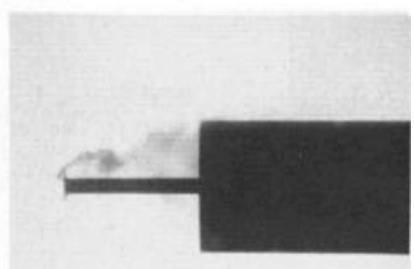

0.75

(0.34)

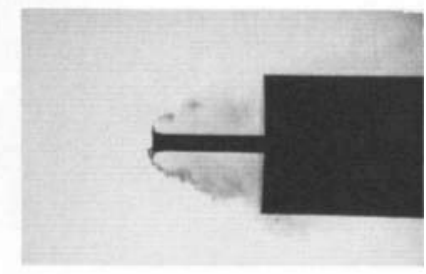

0.5

$(0.42)$

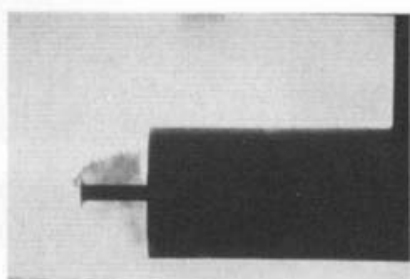

0.25

(0.64)

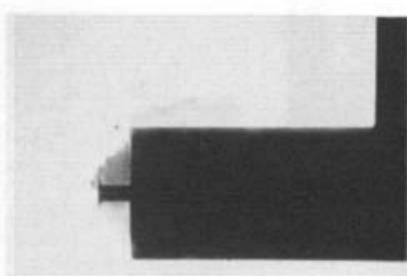

0.125

$(0.71)$

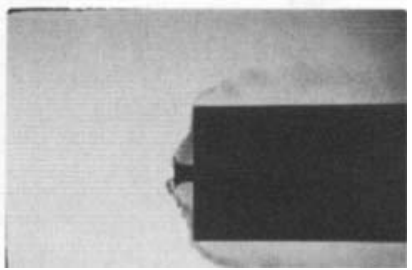

Figure $7(a)$. For description see p. 177. 

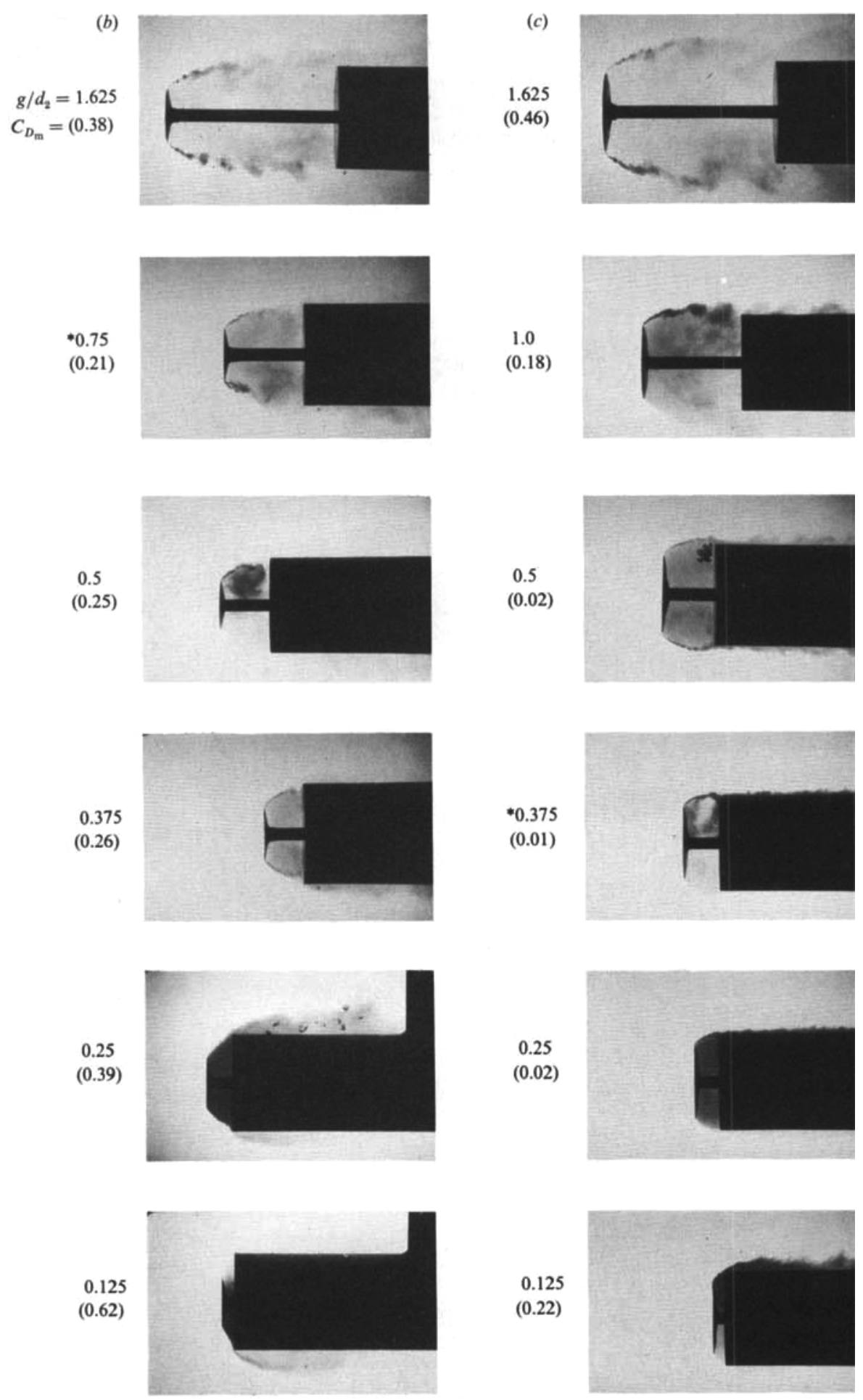

Figure $7(b, c)$. For description see opposite. 

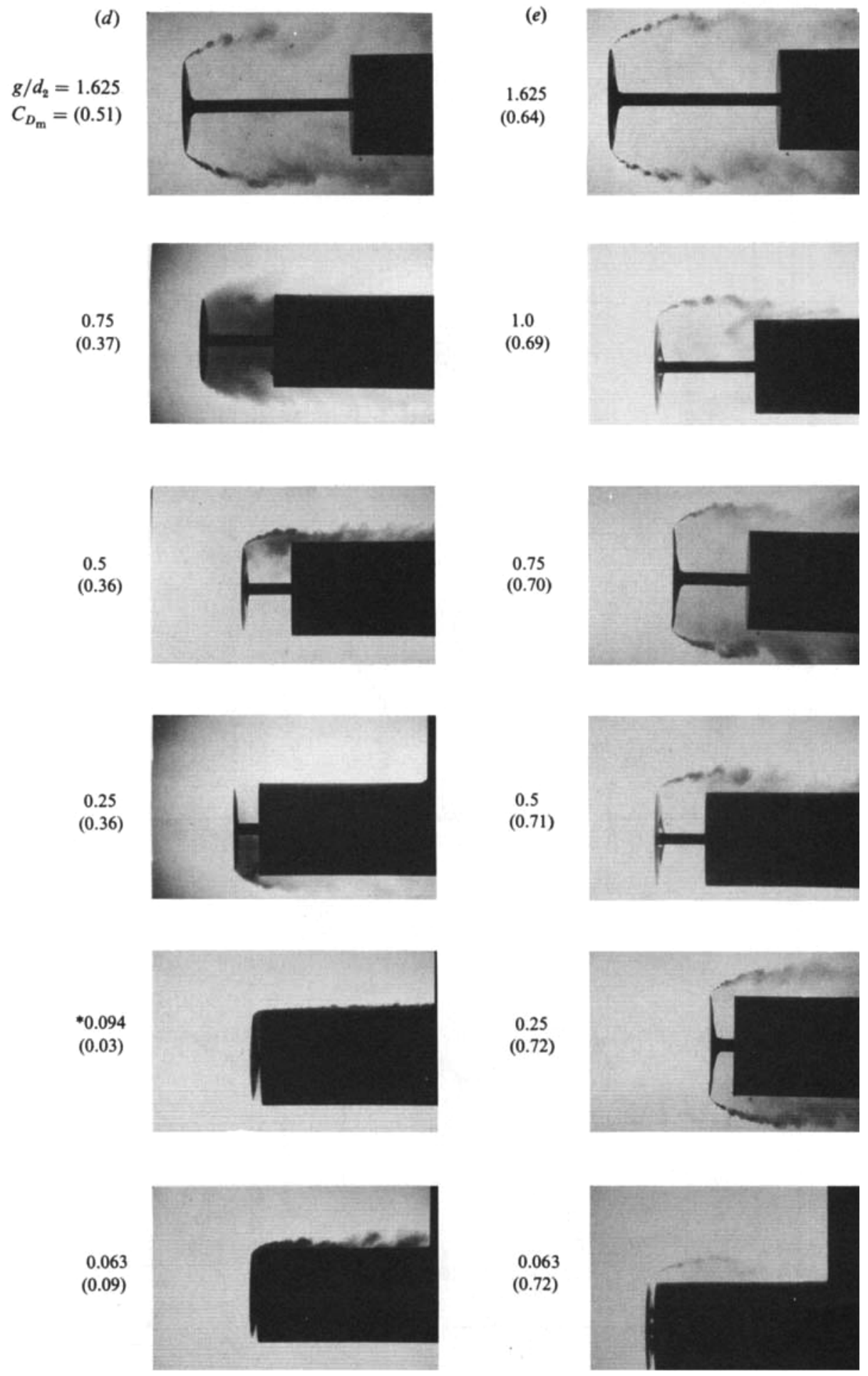

Figure 7. Visuatization of $d_{1} / d_{2} ; *$, optimum gap. (a) $d_{1} / d_{2}=0.25$,

(b) $0.5,(c) 0.75,(d) 0.875,(e) 1.0$. 


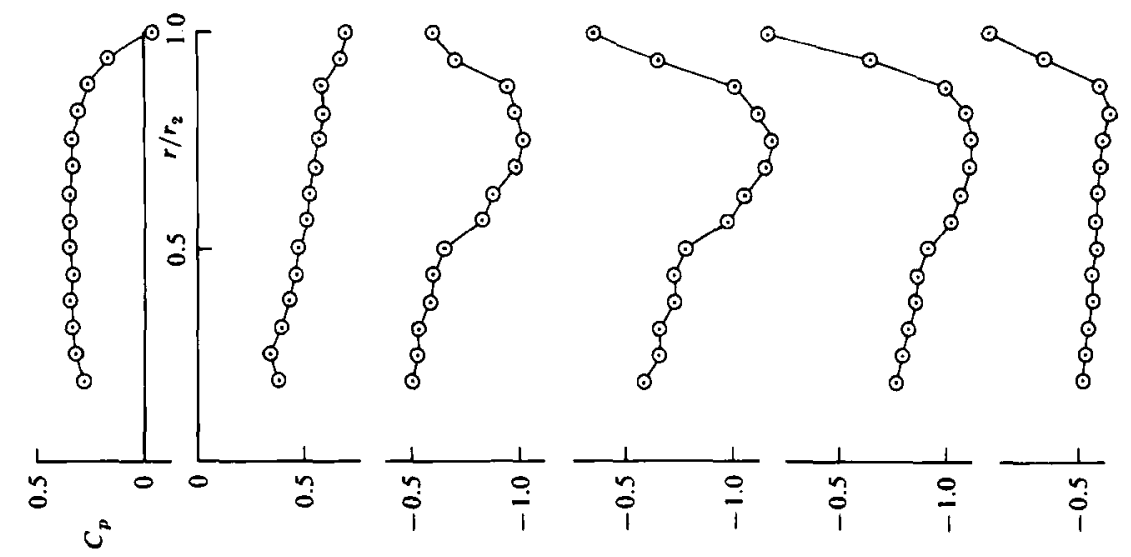

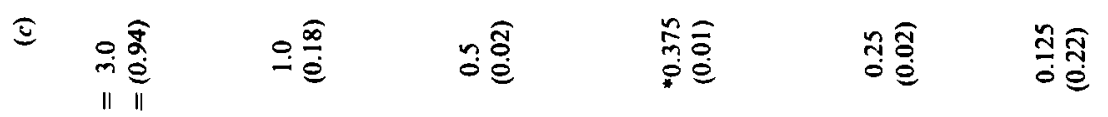
$\frac{s^{N}}{\infty} e^{E}$

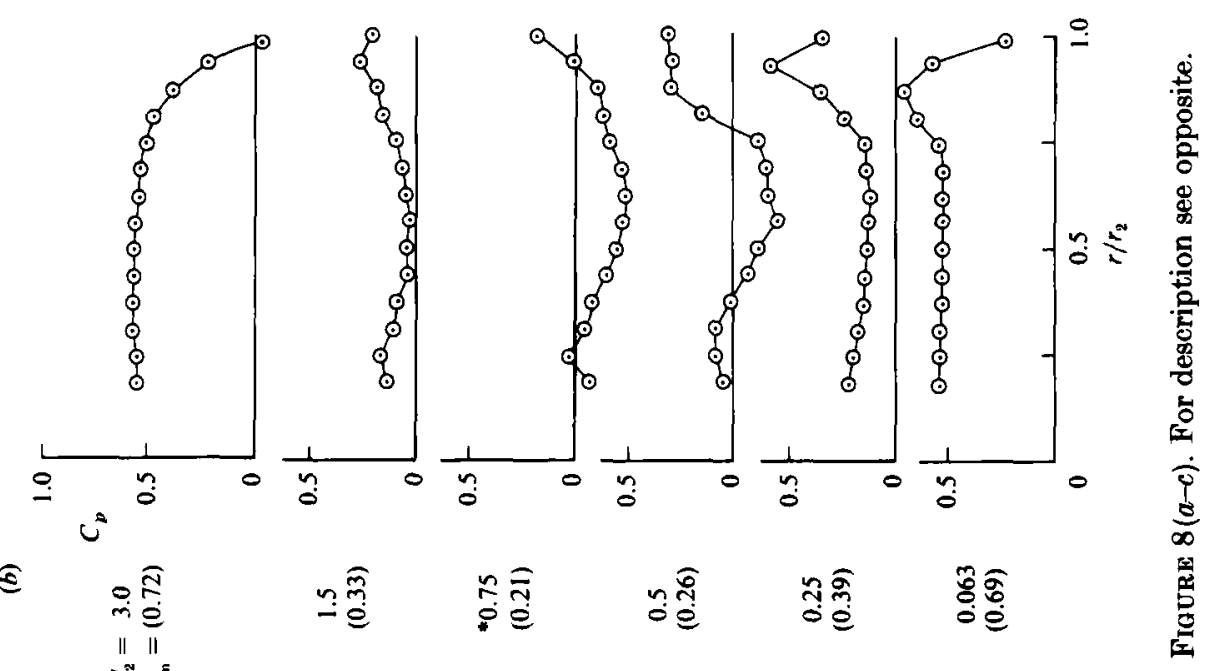

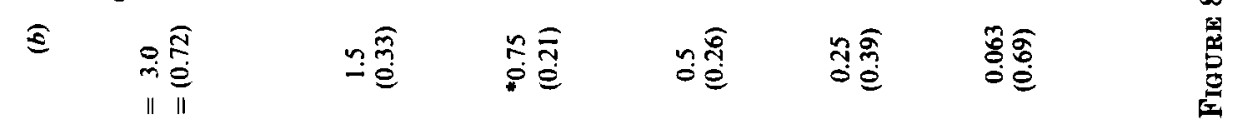
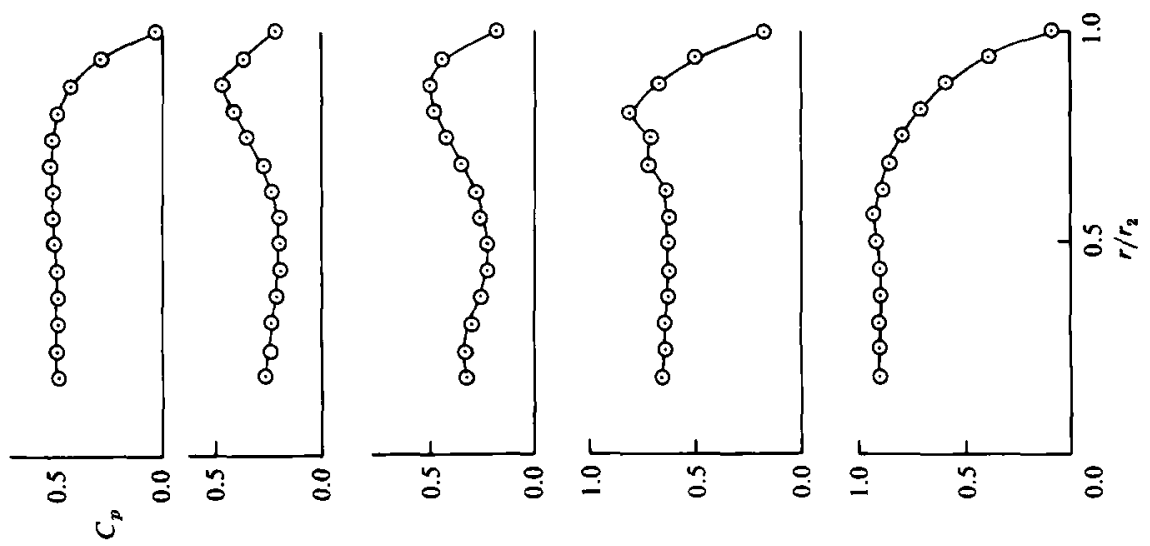

$$
\text { ร) }
$$

䠛

䠛

홍즐 


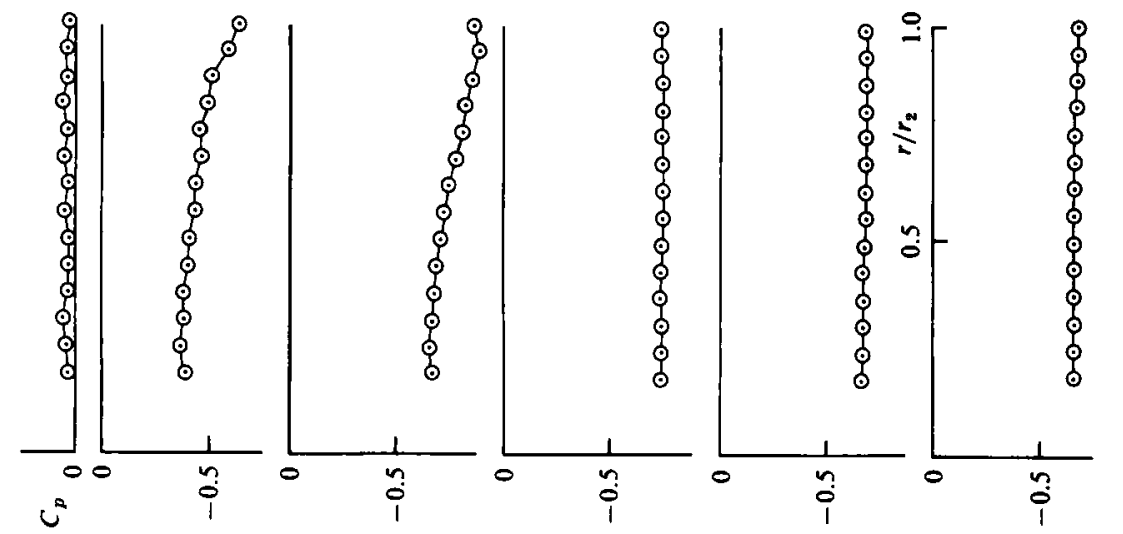

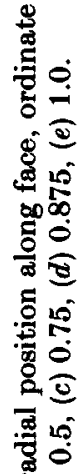

Ð

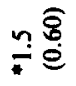

웅

ণุ๊

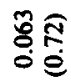

要全

$\frac{x^{2}}{0^{2}}$
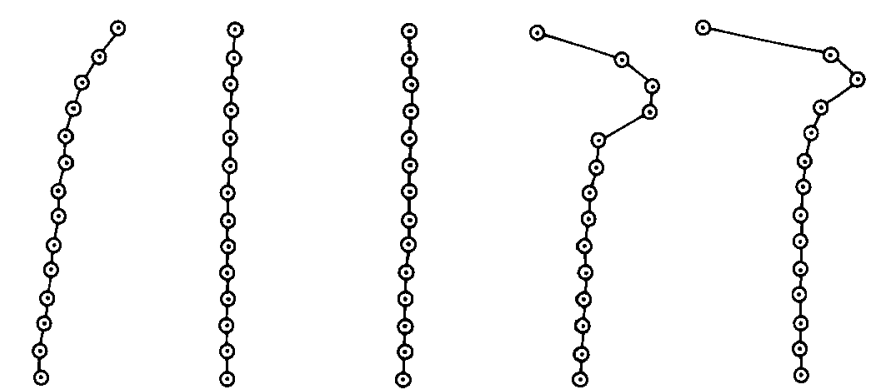

$\frac{1}{\tilde{n}^{2}}$
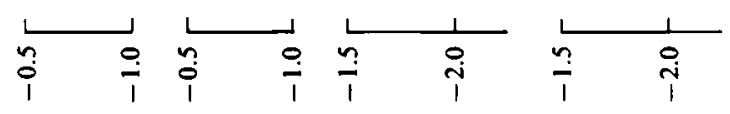

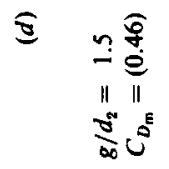

ণุ仓

कํำ

ริ⿳⼈ㄹㅇ

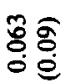

曾

惫

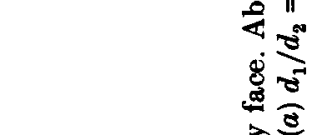


short enough that non-steady aspects of the structure are exhibited. The optimum configurations are indicated by an asterisk (*). In studying these patterns it is instructive to refer to figure 8 , where pressure distributions on the face of the cylinder are plotted. Results for each value of $d_{1} / d_{2}$ will now be discussed separately.

$d_{1} / d_{2}=0.25$. Figure $7(a)$ shows how the disk wake changes as the disk approaches the cylinder. For large gaps, reattachment is onto the sting but at $g / d_{2}=0.75$ (i.e. $\left.g / d_{1}=3.0\right)$ the wake has opened up and reattachment is onto the cylinder face. Pressure distributions in figure 8(a) show how the disk wake depresses the pressure over the central part of the cylinder face for configurations near optimum. But when the disk is very close to the cylinder face it has little effect on the pressure distribution.

$d_{1} / d_{2}=0.5$. At conditions near optimum $\left(g / d_{2}=0.75\right.$ in figure $\left.7 b\right)$ the separated shear layer from the disk attaches at or near the shoulder of the cylinder, but there appears to be considerable unsteadiness in the shear layer. Pressures on the face of the cylinder have become negative (figure $8 b)$. For smaller gaps $\left(g / d_{2}=0.375\right)$ the shear layer is steady and very thin and reattachment is on the face of the cylinder, with correspondingly higher pressure there. For large gap $\left(g / d_{2}=1.625\right)$ large structure can be seen in the free shear layer, which again seems to be reattaching at or near the shoulder of the cylinder.

$d_{1} / d_{2}=0.75$. Qualitatively the flows are similar to those at $d_{1} / d_{2}=0.5$ but at and near optimum conditions the values of $C_{D}$ are very much lower. It may be seen from the flow pictures (figure $7 \mathrm{c}$ ) that the free shear layers are very thin and reattach onto the cylinder shoulder. The boundary layer on the sides of the cylinder is also thin. The pressure coefficient (figure $8 c$ ) is negative over all the cylinder face and has an interesting minimum near the outer part. The contrast between the cases $g / d_{2}=0.5$ and $g / d_{2}=1.0$ should be noted. For the latter the shear layer contains large structures and grows much more rapidly; $C_{D}$ is an order of magnitude larger.

$d_{1} / d_{2}=0.875$. Very low values of $C_{D}$ occur only for very low values of the gap. The corresponding optimum flows (figure $7 d$ ) have the same qualitative features as for $d_{1} / d_{2}=0.75$. The pressure minimum near the outer part of the cylinder face (figure $8 d$ ) is very prominent. At larger but still small gaps, for which $C_{D}$ is an order of magnitude higher, the shear layer is tending to reattach onto the sides of the cylinder. It is qualitatively similar to the flows in the next category.

$d_{1} / d_{2}=1.0$. (See figure 7 e.) For small gaps, reattachment is onto the side of the cylinder but for $g / d_{2}=1.0$ and 1.625 there is a tendency for reattachment onto the edge. The optimum condition occurs within this range, at $g / d_{2}=1.5$ (flow picture not obtained). Except near optimum, the pressure distribution on the cylinder face (figure $8 e$ ) tends to be quite uniform.

At the maximum gap, $g / d_{2}=3$, attained in these experiments, the interaction for all disk diameters is still strong. For $g / d_{2} \rightarrow \infty$ (and if there were no sting present), the asymptotic forebody-system drag would be the sum of the forces on a free disk and on the free face of the cylinder. Normalized with respect to $d_{2}$, the freestream value would be

$$
C_{D} \rightarrow C_{D_{2}}+C_{D_{1}}\left(\frac{d_{1}}{d_{2}}\right)^{2}=0.75+1.12\left(\frac{d_{1}}{d_{2}}\right)^{2},
$$

where we have used for the disk the freestream value 1.12 (Morel \& Bohn 1980). For $d_{1} / d_{2}=0.5$, as an example, the resulting asymptotic value would be $C_{D}=1.03$. In figure 4 , at $g / d_{2}=3$ and $d_{1} / d_{2}=0.5$ the value of $C_{D}$ is still far from that value. 


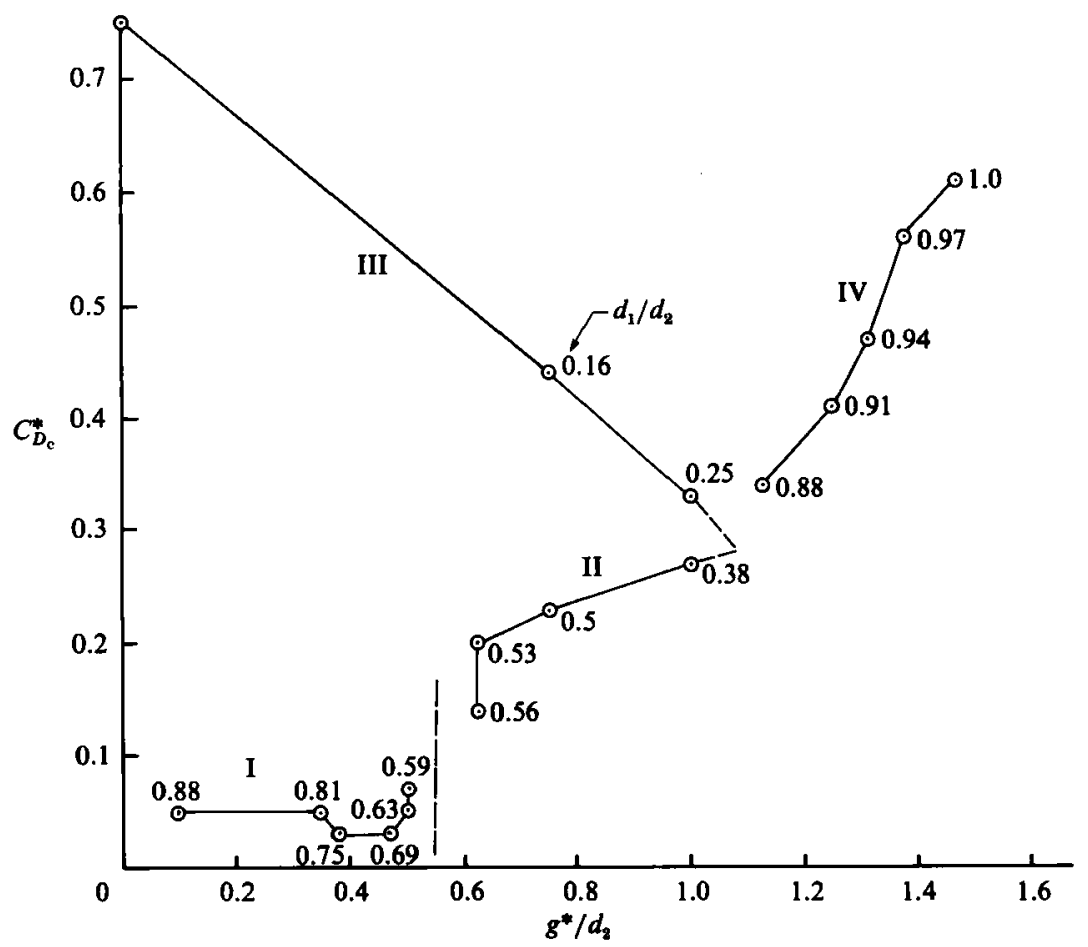

Figure 9. The corrected minimum drag coefficient for each disk and the gap at which it occurs. The value of $d_{1} / d_{2}$ is shown beside each plotted point. Roman numerals refer to flow regimes.

\section{Drag regimes based on optimum flows}

In figure 9 the values of $C_{D_{\mathrm{c}}}^{*}$ for each value of $d_{1} / d_{2}$ from figure 5 have been plotted against the corresponding optimum gap ratio $g^{*} / d_{2}$. The values of $d_{1}^{*} / d_{1}$ are noted beside each point. The points group themselves onto four branches that have been selected as shown with the help of figure 10, where the data are plotted in the $\left(g, d_{1}\right)$-plane. Also use was made of the fact that one of the points must correspond to $C_{D_{\mathrm{c}}}^{*}=0.75$ at $g^{*} / d_{2}=0$.

One of the branches (I) will be called the low-drag branch $\left(C_{D_{\mathrm{c}}}^{*}<0.1\right)$, II is a medium-drag branch $\left(0.1<C_{D_{\mathrm{c}}}^{*}<0.3\right)$ and III and IV will be called high-drag branches $\left(C_{D_{\mathrm{c}}}^{*}>0.3\right)$. Table 1 lists the corresponding ranges of $d_{1}^{*} / d_{2}$ and $g^{*} / d_{2}$. In figures 9 and 10 , note the jump from branch I to IV at $d_{1}^{*} / d_{2}=0.88$. In figure 10, the lowand medium-drag branches pass close to the free-streamline locus, which is discussed in the following section. This locus passes close to the triple point BCD of figure 2. The uncertainty bar on the point for $d_{1} / d_{2}=0.813$ is to draw attention to the flatness of the minimum in figure 5 . Shorter bars on other points are omitted.

This classification of the optimum drag coefficients corresponds to distinctively different flow types. In the high-drag regime III, the disk is too small to guide the separated flow onto the shoulder of the cylinder; in the high-drag regime IV, the disk is too close to the cylinder; in the low-drag regime $I$, the disk is in a range where the separation streamline can reattach at or near the shoulder, in a cavity configuration close to the free-streamline ideal; the medium-drag regime II is a less stable counterpart of I, less stable because the gap is too large. 


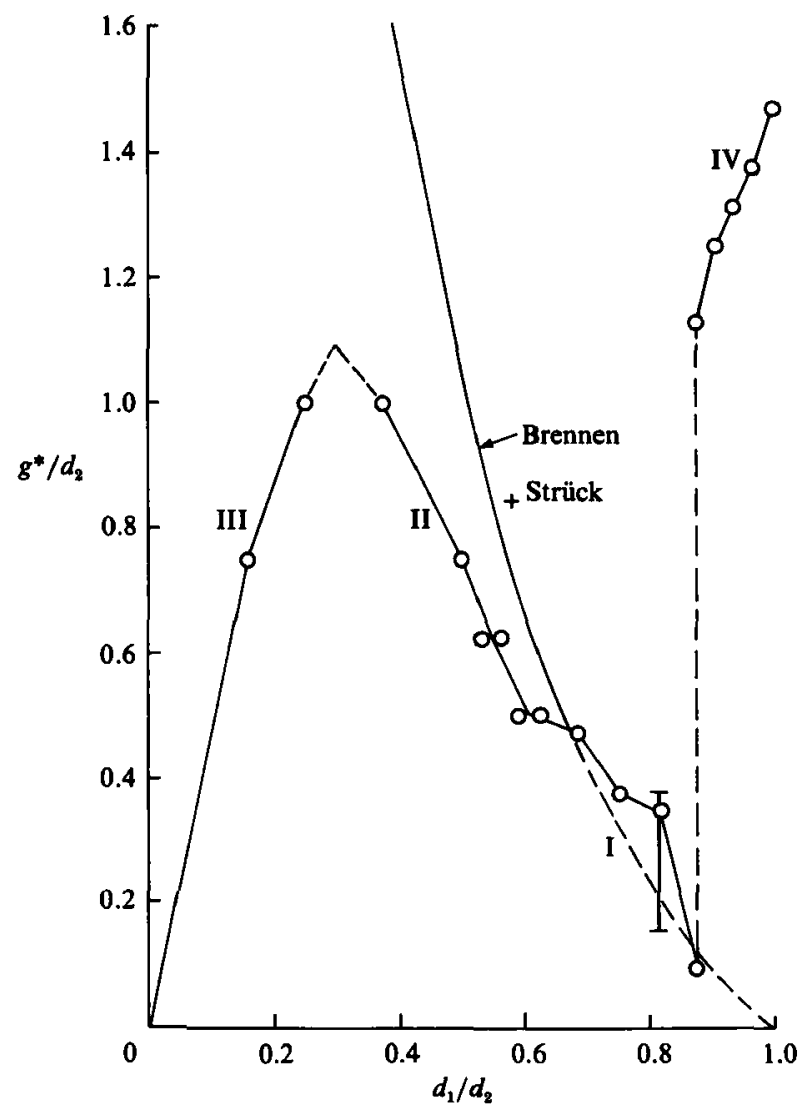

Figure 10. The optimum configurations: $O$, present measurements, the bar indicates uncertainty in identifying $g^{*} / d_{2}$ for this disk (see figure $5 b$ ); continuous curve is the axisymmetric free-streamline result of Brennen (1969); + , axisymmetric free-streamline result of Strück (1970).

\begin{tabular}{ccccc}
\hline Regime $\ldots$ & I & II & III & IV \\
$C_{D_{c}}^{*}$ & Low & Medium & High & High \\
& $<0.1$ & $0.1-0.3$ & $0.75-0.3$ & $>0.3$ \\
$d_{1}^{*} / d_{2}$ & $0.56-0.88$ & $0.3-0.56$ & $0-0.3$ & $>0.88$ \\
$g^{*} / d_{2}$ & $0.094-0.6$ & $0.6-1.1$ & $0-1.1$ & $>1.1$ \\
& & TABLE 1. & Optimum-drag regimes \\
\hline
\end{tabular}

\section{Free-streamline model}

As may be seen in figure 7, the flow patterns for optimum configurations on the low-drag branch are similar to those postulated for the triple-point configuration BCD in figure 2, with the separation streamline from the edge of the disk joining onto the rearbody at the shoulder. If the pressure within the cavity formed by the disk and the cylinder face could be assumed uniform this would be a free-streamline flow, like that sketched in figure 11, with constant pressure along the free-stream surface. The 


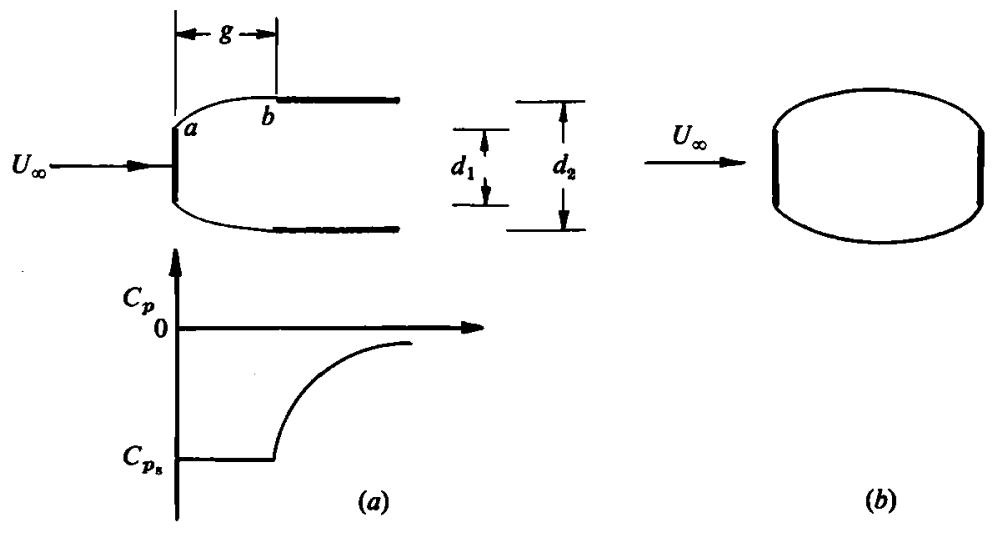

Figure 11. Schematic diagram of free-streamline models. (a) Depicts the disk-cylinder Joukowsky (1890) model and the free-streamline pressure. $(b)$ is the Riabouchinsky (1920) model.

free streamline springing from $a$ at the edge of the disk (or flat plate, in two-dimensional flow) is at constant pressure $p_{\mathbf{s}}$ up to the point $b$, the edge of the rearbody. The cavity pressure coefficient

$$
C_{p_{\mathrm{s}}}=\frac{p_{\mathrm{s}}-p_{\infty}}{\frac{1}{2} \rho U_{\infty}^{2}}=1-\left(\frac{U_{\mathrm{s}}}{U_{\infty}}\right)^{2} \leqslant 0,
$$

where $U_{\mathrm{s}}$ is the constant value of flow speed along the free streamline.

The solution to the problem of finding the free-streamline shape is well known for the two-dimensional case; it was first found by Joukowsky (1890) using mapping techniques (also see Roshko 1954). For the axisymmetric case, numerical techniques have to be used; the result for one value of $C_{p_{s}}$ has been obtained by Strück (1970). A closely related solution is that of Riabouchinsky (1920) for the free-streamline cavity flow between two flat plates (figure $11 b$ ). With disks instead of flat plates, the corresponding axisymmetric solution was calculated numerically by Brennen (1969). We have made use of the latter results by assuming that the shapes of the free streamlines in the Joukowsky flow are very nearly the same as in the front half of the Riabouchinsky flow. Brennen's calculations, for values of $d_{1} / d_{2}$ up to 0.675 , have been extrapolated to $d_{1} / d_{2}=1, g / d_{2}=0$ as shown in figure 10 . Also shown is the single point calculated by Strück; it gives some indication of the adequacy of using the front half of the Riabouchinsky configuration to represent the disk-cylinder model.

The experimental low-drag branch I lies close to the theoretical, free-streamline geometry; the medium-drag branch II is a little below.

The free-streamline pressure coefficient $C_{p_{\mathrm{s}}}$ is plotted against $d_{1} / d_{2}$ in figure 12 . To obtain an estimate for values of $d_{1} / d_{2}$ larger than those computed by Brennen, an approximate formula was developed by making use of a semi-empirical relation by Garabedian (1956) for the drag of an isolated disk with a cavity wake, namely

$$
C_{D_{1}}=0.827\left(1-C_{p_{\mathrm{s}}}\right)
$$

where $C_{D_{1}}$ is the drag coefficient of the disk based on its own diameter. Then, following an idea by Eppler (1956), the constant-pressure, free-stream surface can be regarded as part of a solid body formed with the disk and the rearbody. The drag of such a 


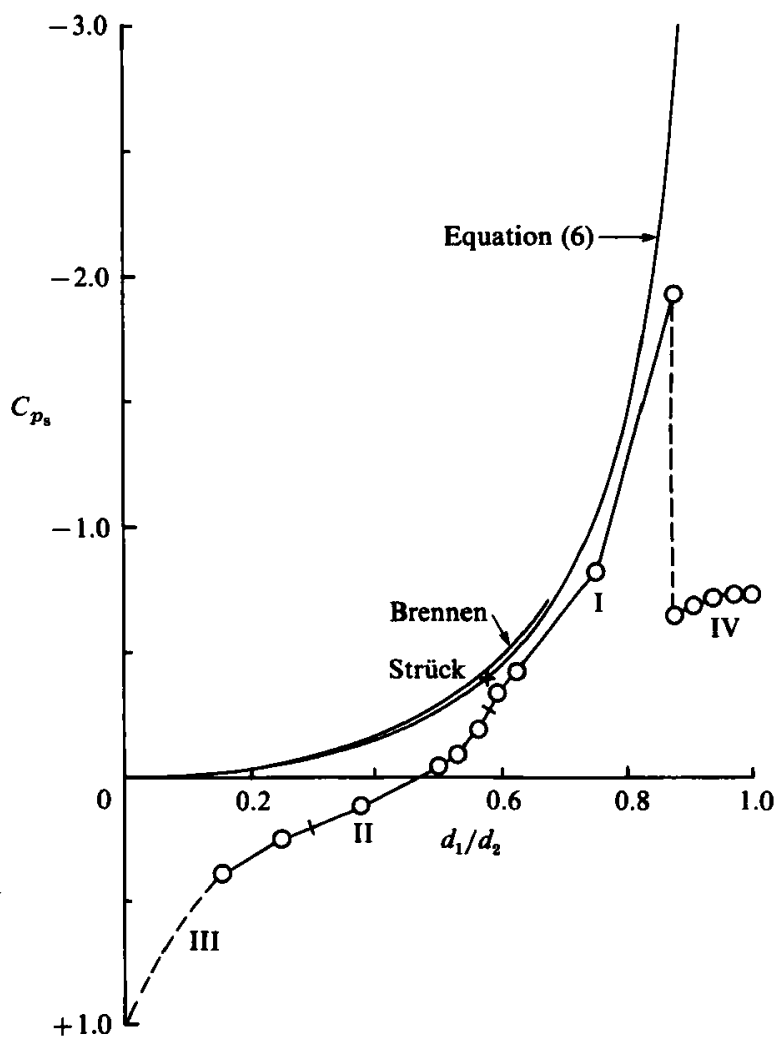

FIGURe 12. Axisymmetric, free-streamline pressure coefficients: truncated continuous curve is from Brennen (1969); extended continuous curve is the interpolation formula, equation (6); + , Strück (1970); $O$, representative cavity-pressure coefficients from present measurements.

semi-infinite body in unbounded potential flow is zero (Prandtl \& Tietjens 1934), i.e.

$$
\begin{aligned}
0=D & =\int\left(p-p_{\infty}\right) \mathrm{d} A, \quad A=\text { projected frontal area } \\
& =\left(\bar{p}_{1 f}-p_{\infty}\right) A_{1}+\left(p_{\mathrm{s}}-p_{\infty}\right)\left(A_{2}-A_{1}\right),
\end{aligned}
$$

where $\left(\bar{p}_{1 \mathrm{f}}-p_{\infty}\right) A_{1}=\bar{C}_{p_{\mathrm{q}}} q_{\infty} A_{1}$ is the contribution from the front face of the body and $\left(p_{\mathrm{s}}-p_{\infty}\right)\left(A_{2}-A_{1}\right)=C_{p_{\mathrm{s}}} q_{\infty}\left(A_{2}-A_{1}\right)$ is from the constant-pressure part of the surface. This gives a relation between the free-streamline pressure coefficient and the front-face force,

$$
-C_{p_{\mathrm{s}}}\left(A_{2}-A_{1}\right)=\bar{C}_{p_{\mathrm{f}}} A_{1} .
$$

On the other hand, the drag of a disk may be written

$$
C_{D_{1}}=\bar{C}_{p_{\mathbf{f}}}-C_{p_{\mathrm{s}}}
$$

where $C_{p_{\mathrm{s}}}$ is now the base pressure coefficient for the disk. With the reasonable assumption that, for given base pressure, the disk in the cavity configuration experiences very nearly the same force as when isolated, the above set of equations can be solved to find

$$
C_{p_{\mathrm{s}}}=\frac{-0.827}{\left(d_{2} / d_{1}\right)^{2}-0.827}
$$


This formula will evidently not be correct for $d_{1} / d_{2} \rightarrow 1$ for which, according to the free-streamline model, $C_{p_{\mathrm{s}}} \rightarrow-\infty$. Thus the alternative interpolation formula,

$$
C_{p_{\mathrm{s}}}=\frac{-0.827}{\left(d_{2} / d_{1}\right)^{2}-1}
$$

which has the right form for $d_{2} / d_{1} \rightarrow 0$ as well as for $d_{2} / d_{1} \rightarrow 1$, seems more appropriate. As shown in figure 12, it agrees well with the numerical calculations of Strück for the Joukowsky model and of Brennen for the Riabouchinsky model.

Also plotted in figure 12 are experimental values of $C_{p_{3}}$ which have been chosen to be representative of the cavity pressures in the experimental configurations. Choosing these values is of course problematic since pressure is not in fact uniform throughout the separated region because it is a dynamic region, albeit at low dynamic pressures. Since pressure measurements were made on the cylinder face (figure 8) and not on every disk base, the former were used together with the overall drag measurement first to compute $C_{D_{1}}$ and then to deduce the average disk base pressure from (2). These are the values which are plotted in figure 12. In the low-drag range (I) they lie quite close to the free-streamline model calculations; in the medium-drag range (II) $C_{p_{\mathbf{s}}}$ departs considerably from the theoretical values and even becomes positive.

\section{A model for drag at optimum conditions}

The preceding comparison of experimental results with parameters from the free-streamline model indicates that good correspondence occurs for the configuration on the low-drag branch. This suggests the possibility of estimating the drag of these configurations by replacing the free streamline in that model by a real shear layer. The procedure we use can be illustrated by reference to the problem of two-dimensional flow over a cutout in a wall which is parallel to a free stream (figure 13a). In this case the free streamline is simply the straight line $a b$ and the drag of the cavity is zero. In the real flow there is a drag force $D$, which may be obtained from the momentum balance on the fluid enclosed by contour abcda; it is the negative of the net force on the cavity walls, i.e.

$$
D=\int_{a}^{b} \tau_{\mathbf{g}} \mathrm{d} x=\int_{a}^{b}\left(-\rho \overline{u^{\prime} v^{\prime}}-\rho \overline{u v}\right) \mathrm{d} x,
$$

where $-\rho \overline{u^{\prime} v^{\prime}}$ is the Reynolds stress along $a b$ and $-\rho \overline{u v}$ is the mean momentum transport, which is not zero because the dividing streamline in general does not coincide with $a b$ and thus $\bar{v}$ is not zero (Gharib 1983). Equation (7) may be written

$$
D=C_{\tau} \rho U_{\infty}^{2} g,
$$

where $C_{\tau}$ is the mean value (over the gap) of the generalized shear stress,

$$
\tau_{\mathrm{g}} \equiv-\rho \overline{u^{\prime} v^{\prime}}-\rho \overline{u v}
$$

normalized by $\rho U_{\infty}^{2}$.

If the sketch in figure $13(a)$ is taken to be a meridional section of an axisymmetric cylinder, as in Gharib's experiment, then $D=C_{\tau} \rho U_{\infty}^{2} \pi d_{2} g$. With $C_{D}$ based on frontal area,

$$
C_{D}=\frac{8 C_{\tau} g}{d_{2}}
$$




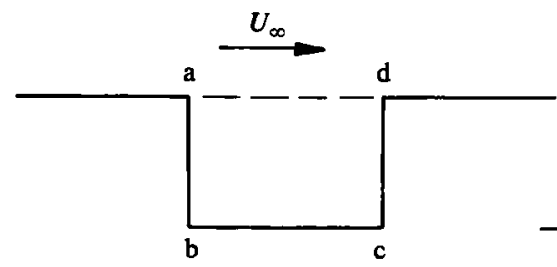

(a)

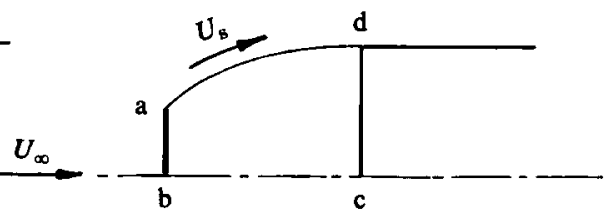

(b)

FIGURE 13. Schematic diagram of cavity flow models. (a) A cutout in a wall; (b) represents the present disk-cylinder geometry.

This result is applicable to the case in which the frontbody in our configuration is a hemisphere, discussed in a later section.

To apply these ideas to the present experiments on the disk-cylinder configuration, those flows which lie close to the free-streamline trajectory in figure 10 may also be viewed as cavity flows (figure $13 b$ ). The cavity drag and hence the forebody drag (assuming no change in $\bar{C}_{p_{f}}$ ) is then

$$
D=2 \pi \int_{a}^{b} \tau_{\mathrm{g}} r_{\mathrm{s}} n_{x} \cdot \mathrm{d} s=2 \pi \int_{0}^{g} \tau_{\mathrm{g}} r_{\mathrm{s}} \mathrm{d} x,
$$

where $r_{\mathrm{s}}(x)$ is the free streamline boundary and $\mathrm{d} x$ is the $x$ component of the vector element $\mathrm{d} s$ along the free streamline. For given cavity pressure $C_{p_{\mathrm{s}}}$ the pressure on the front of the disk and along the dividing streamline is assumed to be the same as in the free-streamline model. The appropriate reference for $\tau_{\mathrm{g}}$ is now the velocity on the free streamline, i.e.

$$
\tau_{\mathrm{g}}=C_{\tau} \rho U_{\mathrm{s}}^{2}
$$

and noting that now $U_{\mathrm{s}}=\left(1-C_{p_{\mathrm{s}}}\right)^{\frac{1}{2}} U_{\infty}$, we obtain

$$
D=2 \pi C_{\tau} \rho U_{\infty}^{2}\left(1-C_{p_{\mathrm{s}}}\right) \int_{0}^{g} r_{\mathrm{s}} \mathrm{d} x .
$$

The integral could be calculated numerically from the free-streamline results for the various optimum geometries. Instead, in view of the various assumptions already made, it seems sufficient to approximate it by an average value $r_{\mathrm{s}}=\frac{1}{2}\left(r_{1}+r_{2}\right)=\frac{1}{4}\left(d_{1}+d_{2}\right)$, which is equivalent to approximating the area of the free surface by that of a truncated cone. This gives then

$$
D=\frac{1}{2} \pi C_{\tau} \rho U_{\infty}^{2}\left(1-C_{p_{\mathrm{s}}}\right)\left(d_{1}+d_{2}\right) g,
$$

and

$$
C_{D}=4 C_{\tau}\left(1-C_{p_{\mathrm{s}}}\right)\left(1+\frac{d_{1}}{d_{2}}\right) \frac{g}{d_{2}}
$$

Next, (6) is used to write $C_{p_{\mathrm{s}}}$ as a function of $d_{1} / d_{2}$ and then the numerical results of Brennen, as shown in figure 12, are used to obtain a relation between $d_{1} / d_{2}$ and $g / d_{2}$. Thus $C_{D}$ can be obtained as a function of either $g / d_{2}$ or $d_{1} / d_{2}$, with $C_{\tau}$ as a parameter.

With $g / d_{2}$ as the variable the formula could be plotted in figure 9 but instead we present the data in figure 14, where $d_{1} / d_{2}$ is used as the variable. Here, the measured values of optimal $C_{D}$, corrected for effects of blockage and extraneous skin friction as explained in the Appendix, are compared with the prediction of $(10 c)$ for three 


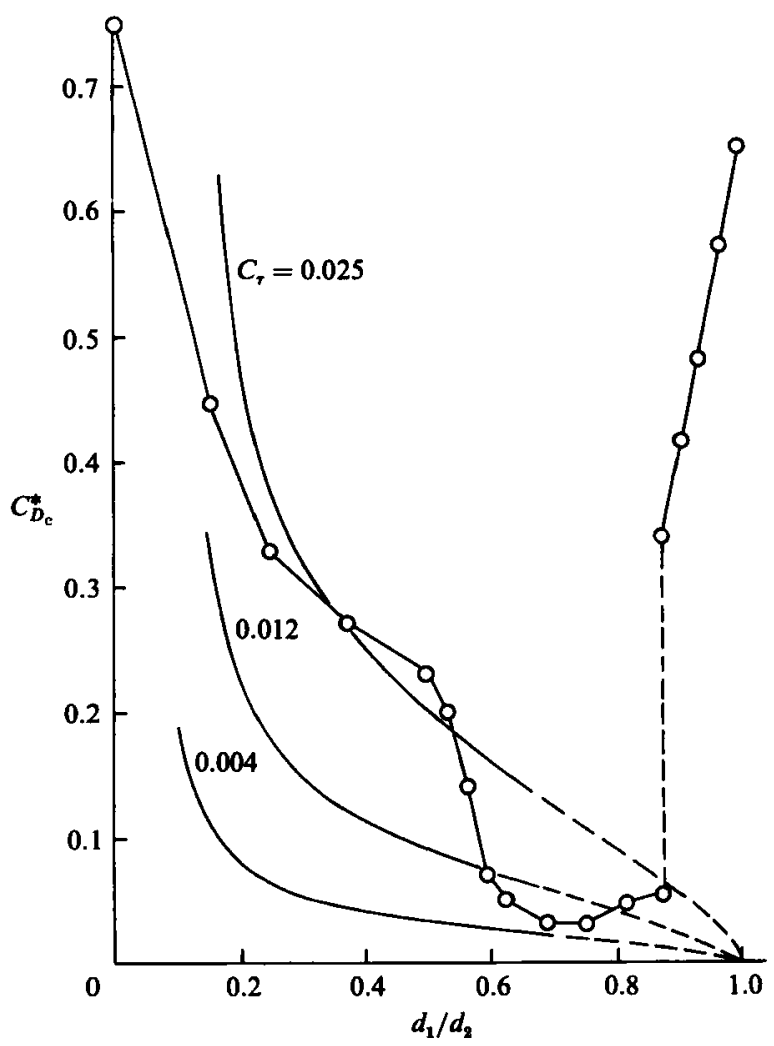

Figure 14. Drag at optimum conditions: $O$, corrected measurements of the minimum-drag coefficient for each disk; - $\longrightarrow$, estimates of forebody drag from equation (10 $b$ ) for different values of $C_{r}$.

values of $C_{\tau}$. One value $\left(C_{\tau}=\mathbf{0 . 0 1 2}\right)$ is typical of Reynolds stress in a plane turbulent free shear layer (e.g. Liepmann \& Laufer 1947; Wygnanski \& Fiedler 1970), while the lower (0.004) and higher $(0.025)$ values are typical of values measured in our low-drag and medium-drag regimes, respectively, as described in the following section.

\section{Flow-field measurements}

The results presented in preceding sections indicate that, corresponding to the quantitative differences in drag, there are fundamental differences in flow properties in the different regimes. To obtain further insight into these, and to obtain some measurements of the Reynolds stresses, we undertook some limited $\dagger$ measurements of flow velocities in the low-drag and medium-drag regimes.

The velocity field between the forebody and rearbody was investigated with a single-particle laser-Doppler velocimeter, operated in the dual-scatter mode. These measurements were made in the GALCIT free-surface water tunnel using the flow-visualization model. The Reynolds number was fixed at $1 \times 10^{5}$ based on $d_{2}$. The velocimeter, designed and built especially for this experiment under the guidance of Dr P. E. Dimotakis (Koenig 1978), provided measurements of a single, but selectable, 
velocity component. A special feature of the velocimeter was that each of the two equal-intensity scattering beams (obtained by splitting the single beam from a $5 \mathrm{~mW}$ He-Ne laser) was frequency shifted by a Bragg cell (Intra-Action model ADM-40) with the frequency difference between the beams adjustable from $10 \mathrm{kHz}$ to $10 \mathrm{MHz}$. The wide frequency range available for shifting permitted the velocimeter to be optimized so that there was no directional ambiguity in regions of recirculating or highly turbulent flow while at the same time the loss of accuracy due to the velocity bias was minimized. The forward-scattering light from a particle passing through the overlap volume was focused into a photomultiplier tube which, in turn, provided the input to a counter-type processor (Dimotakis \& Lang 1974). The counter provided the time of flight $t_{i}$ and the number of fringes crossed, $n_{i}$ (which generally was required to be greater than 20), for each acceptable particle passage. From $t_{i}, n_{i}$ and the fringe spacing the corresponding velocity component $u_{i}$ could then be determined. The filtered but unseeded flow yielded a sample rate that was maintained at about 200 samples/s.

We denote the streamwise velocity component by $u$ and the radial component by $v$. The various statistical properties of each component, for example $\bar{u}$, the mean streamwise velocity, and $\overline{u^{\prime 2}}$, the mean square fluctuation in the streamwise direction, were computed from ensemble averages of $u_{i}$, each ensemble consisting of 4000-16000 realizations. The Reynolds stress $-\overline{u^{\prime} v^{\prime}}$ was obtained from measurements at $\pm 45^{\circ}$ to the freestream direction. Because of the fairly low data rate it was necessary to correct the measurements for the sampling bias towards higher-velocity particles (Dimotakis, Collins \& Lang 1978). Corrections were also necessary to account for the velocity gradient across the measurement volume. Details of the measurement system and the data-reduction schemes are more fully described by Koenig (1978).

Samples of measurements obtained from traverses through the cavity on lines passing through the model axis are presented in figures 15-18. These figures display results for one example of a medium-drag geometry, $d_{1} / d_{2}=0.5$, and one example of a low-drag case, $d_{1} / d_{2}=0.75$, at their optimum gaps $g^{*} / d_{2}=0.75$ and 0.375 , respectively. The traverses shown are at one axial location for each configuration, $x / g=0.67$ for the medium-drag geometry, $x / g=0.5$ for the low-drag geometry, with $x$ measured downstream from the plane of the disk.

The mean streamwise-velocity component $\bar{u}$ is compared for the two examples in figure $15(a)$ where straight lines have been used to connect the measurements, omitting the actual data points. Smoothed or interpreted versions of these profiles are presented in figure $15(b)$. For both configurations, in the flow external to the shear layer $\bar{u}$ at first increases from $U_{\infty}$ as the cavity is approached due to the potential-flow acceleration of the fluid as it passes over the body. Indicated on figure $15(b)$ by broken lines are the continuations of these potential-flow profiles as they would appear in a free-streamline flow, based on the nominal cavity pressures. The actual profiles of course are modified by the free shear layers. It may be seen that the free shear layer is much thicker for the medium-drag case than for the low-drag case. The profiles inside each cavity indicate that the mean flow is predominantly a single vortical motion filling the cavity, with the reversed velocity, near the centreline, reaching a maximum of about $0.5 U_{\infty}$ for both cases.

Figures 16 and 17 present profiles of the streamwise $\left(\overline{u^{\prime 2}}\right)^{\frac{1}{2}}$, and radial $\left(\overline{v^{\prime 2}}\right)^{\frac{1}{2}}$, root-mean-square velocity fluctuations, respectively. The medium-drag geometry is characterized by turbulent fluctuations having amplitudes more than twice as great as those occurring in the low-drag configuration. The extent, radially outward, of the turbulence is considerably greater for the medium-drag geometry and the fluctuations 

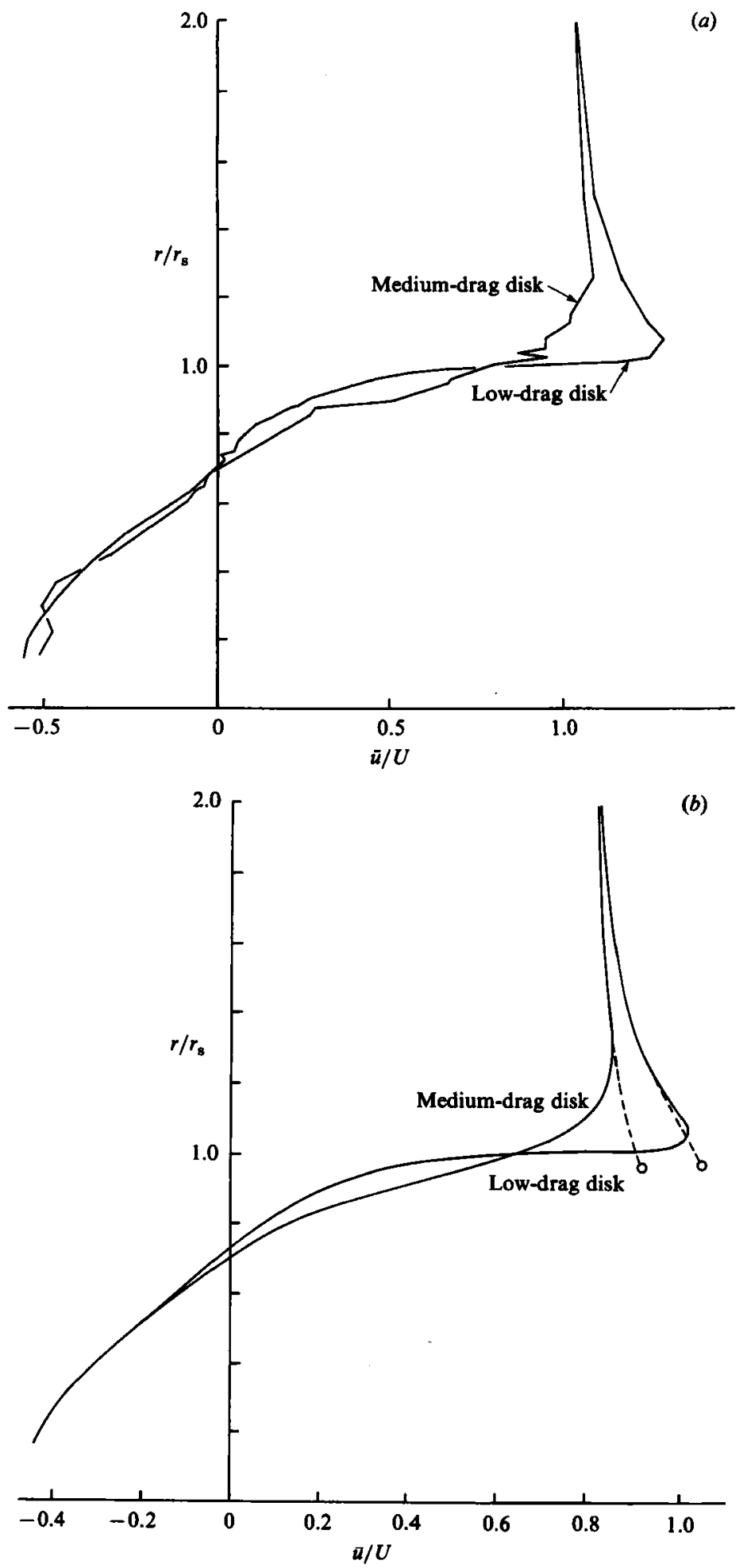

FigURE 15. (a) Mean streamwise-velocity profiles through the cavity for two optimum geometries. The medium-drag disk is $d_{1} / d_{2}=0.5, g^{*} / d_{2}=0.75$ and the measurement station is $x / g^{*}=0.67$; the low-drag disk is $d_{1} / d_{2}=0.75, g^{*} / d_{2}=0.375, x / g^{*}=0.5, x$ measured downstream from the disk. Straight lines connect the data points which have been omitted. $(b)$ The profiles of $(a)$ smoothed. Broken lines represent the potential-flow profiles for free-streamline flow at the nominal cavity pressure. 


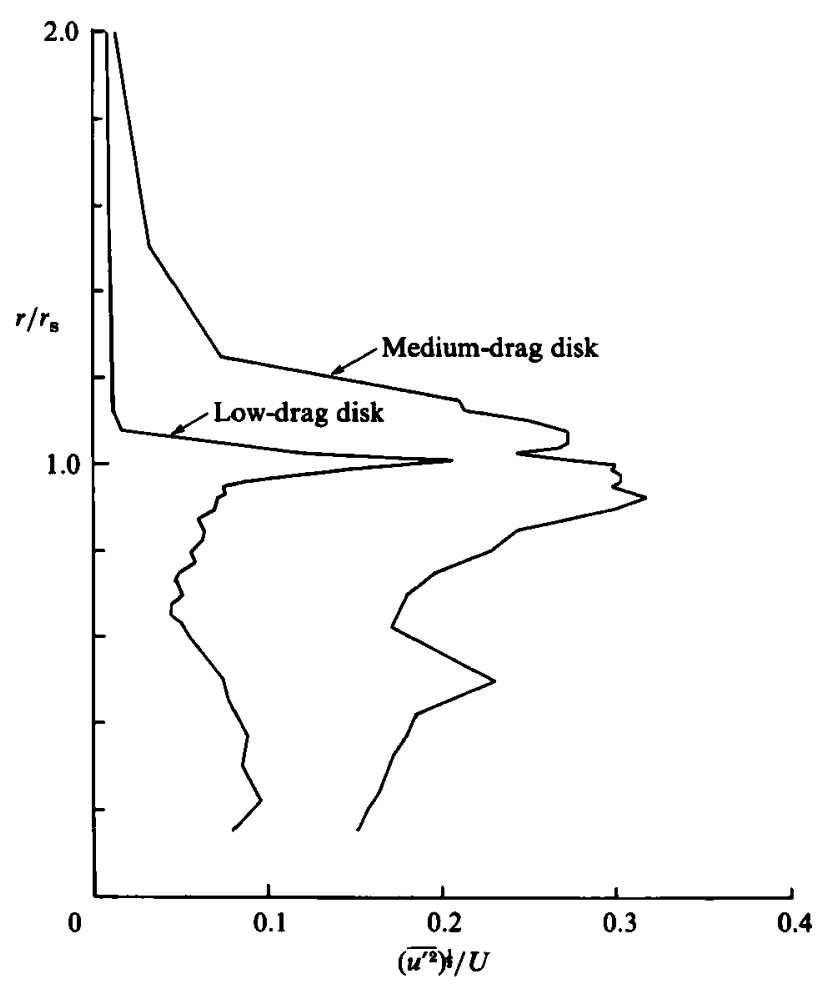

Frgure 16. Root mean-square streamwise-velocity fluctuations through the cavity for the two optimum geometries of figure 15 .

remain large inside its cavity. In the low-drag case the turbulence is quite small midway into the cavity $\left(r / r_{\mathrm{s}}<0.6\right)$ but there is a pronounced increase in $u^{\prime}$ and $v^{\prime}$ near the model centreline. Although figure 15 suggests that the mean flow in each cavity is similar, the profiles of r.m.s. turbulent velocities in figures 16 and 17 clearly show that in detail the flows are quite different. The flow in the low-drag cavity appears to be a fairly well defined and steady 'vortex', easily definable in short-time averages, which perhaps varies slightly, giving rise to the increased fluctuations near the cavity bottom. This behaviour is consistent with that observed in the flow visualization. In the medium-drag flow, on the other hand, the turbulence level is high throughout the cavity and the vortical motion is clearly defined only in longtime averages.

Perhaps the most important difference between the two configurations is revealed by the profiles of streamwise Reynolds stress $-\overline{u^{\prime} v^{\prime}}$ presented in figure 18. The stress in the low-drag flow is very small except for a narrow peak in the shear layer and a small region of reversed stress near the sting. The maximum stress in the low-drag shear layer is 0.004 relative to $\rho U_{\infty}^{2}$. In contrast, in the medium-drag geometry the turbulent stress is high inside the cavity as well as the shear layer, the maximum observed value being 0.023 with respect to $\rho U_{\infty}^{2}$. To compare properly these stresses they should be determined in the local coordinate system of the shear layer and referenced to the maximum velocity just outside the shear layer. When this is done for the profiles presented in figure 18 (details of this transformation are described by Koenig 1978) the maximum tangential turbulent stress coefficient for the low-drag case does not change, so that $\tau_{\mathrm{s}}=0.004 \rho U_{\mathrm{s}}^{2}$, while maximum stress for the medium- 


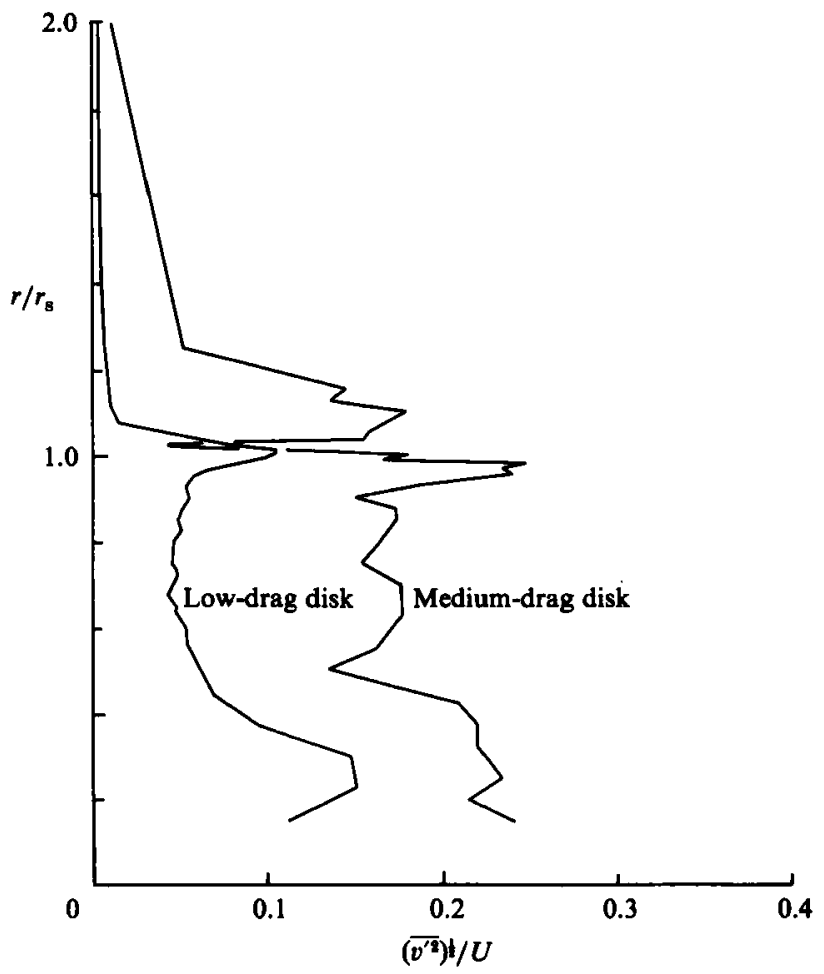

Figure 17. Root mean-square radial-velocity fluctuations through the cavity for the two optimum geometries of figure 15.

drag case becomes $\tau_{\mathrm{s}}=0.025 U_{\mathrm{s}}^{2}$. The coefficients may be compared to the values 0.0115 and 0.016 deduced from the measurements by Liepmann \& Laufer (1947) and by Wygnanski \& Fiedler (1970) respectively, in self-similar, plane free shear layers. The measurements are summarized in table 2 where $\left(-\overline{u^{\prime} v^{\prime}}\right)$ denotes the correlation in the $(x, r)$ coordinate system while $\left(-\overline{u^{\prime} v^{\prime}}\right)_{\mathrm{s}}$ is the value obtained by transforming to the coordinate system of the shear layer at the point of measurement. The latter is the proper one to compare with inferences about $C_{\tau}$ in the model for the drag. It should be emphasized that these values are for only one position in each case, roughly midway along the shear layer; the distribution of shear stress at other points along the shear layer was not determined.

If we take these values to be representative of the mean value $C_{\tau}$ for the whole shear layer, as we have in figure 14, ignoring the fact that the latter may include some component from $(-\overline{u v})$, the numerical agreement with our drag model is surprisingly good. Thus, in figure 14, the curve for $C_{\tau}=0.025$ passes through the upper part of the medium-drag range while the curve for $C_{\tau}=0.004$ is just below the lowest values in the low-drag range. As Gharib (1983) has shown, the variation within the low-drag range can be considerable.

It appears from these comparisons that an important distinction between the low-drag and medium-drag regimes is the marked difference in the turbulence level in the free shear layer which spans the gap, exemplified by the factor of six in the Reynolds stress.

The general nature of the low-drag mean cavity flow is perhaps best seen in the velocity-vector plot depicted in figure 19 . These vectors are determined from profiles 

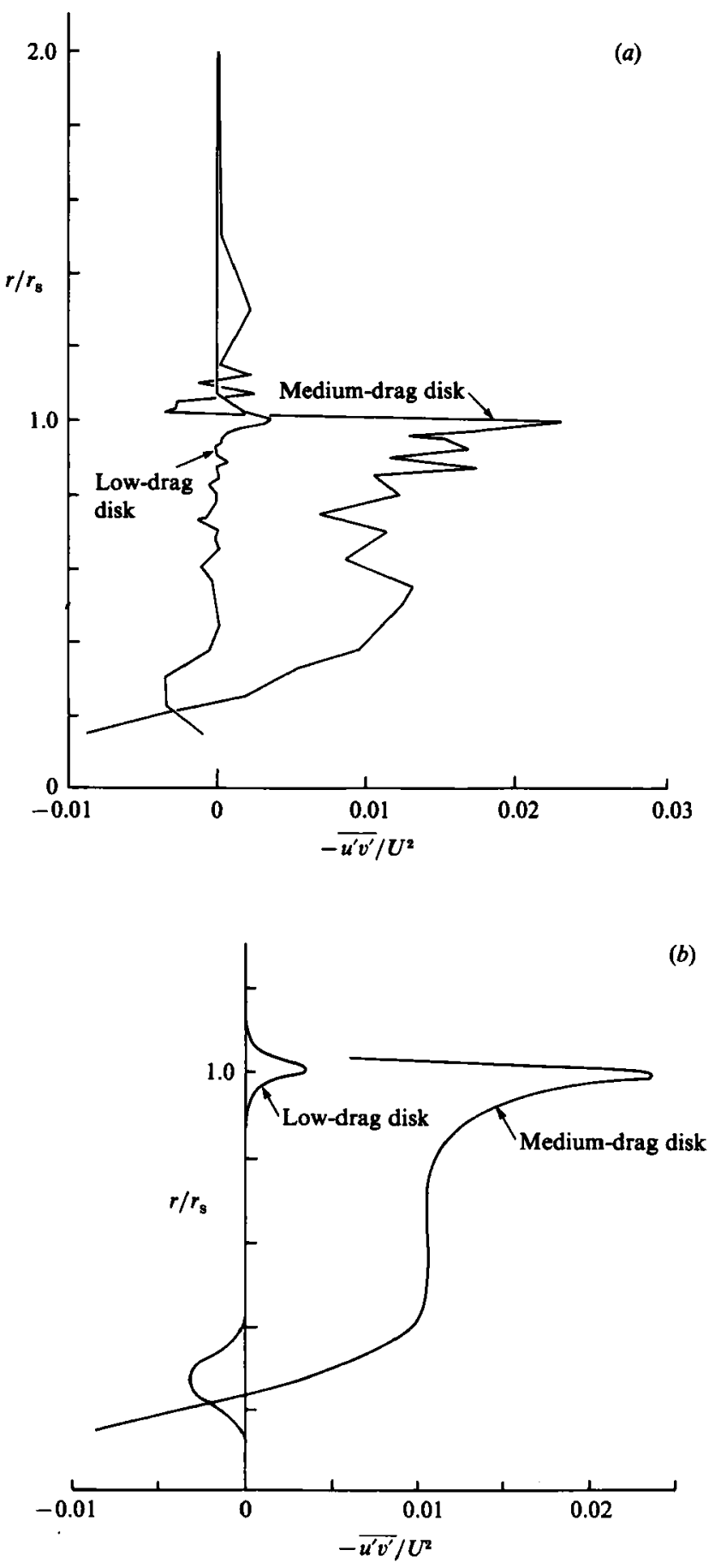

Fraure 18. (a) Streamwise-shear-stress profiles through the cavity for the two optimum geometries of figure 15. (b) The shear-stress profiles of figure $19(a)$ smoothed. 


$\begin{array}{lcccccccc}\text { Configuration } & \frac{x}{g} & \frac{d_{1}}{d_{2}} & \frac{g}{d_{2}} & -C_{p_{\mathrm{s}}} & \frac{U_{\mathrm{s}}}{U_{\infty}} & \frac{-\overline{u^{\prime} v^{\prime}}}{U_{\mathrm{s}}^{2}} & \frac{\left(-\overline{u^{\prime} v^{\prime}}\right)_{\mathrm{s}}}{U_{\mathrm{s}}^{2}} & \frac{-\overline{u^{\prime} v^{\prime}}}{\left(\overline{u^{\prime 2}}\right)^{\frac{1}{2}}\left(\overline{\bar{v}^{2}}\right)^{\frac{1}{2}}} \\ \text { Low drag } & 0.5 & \mathbf{0 . 7 5} & \mathbf{0 . 3 8} & 0.80 & 1.34 & 0.004 & 0.004 & 0.38 \\ \text { Medium drag } & \mathbf{0 . 6 7} & \mathbf{0 . 5 0} & \mathbf{0 . 7 5} & \mathbf{0 . 2 1} & \mathbf{1 . 1 0} & \mathbf{0 . 0 2 3} & 0.025 & \mathbf{0 . 5 2}\end{array}$

TABLE 2. Maximum values of Reynolds stress at the noted values of $x / g$

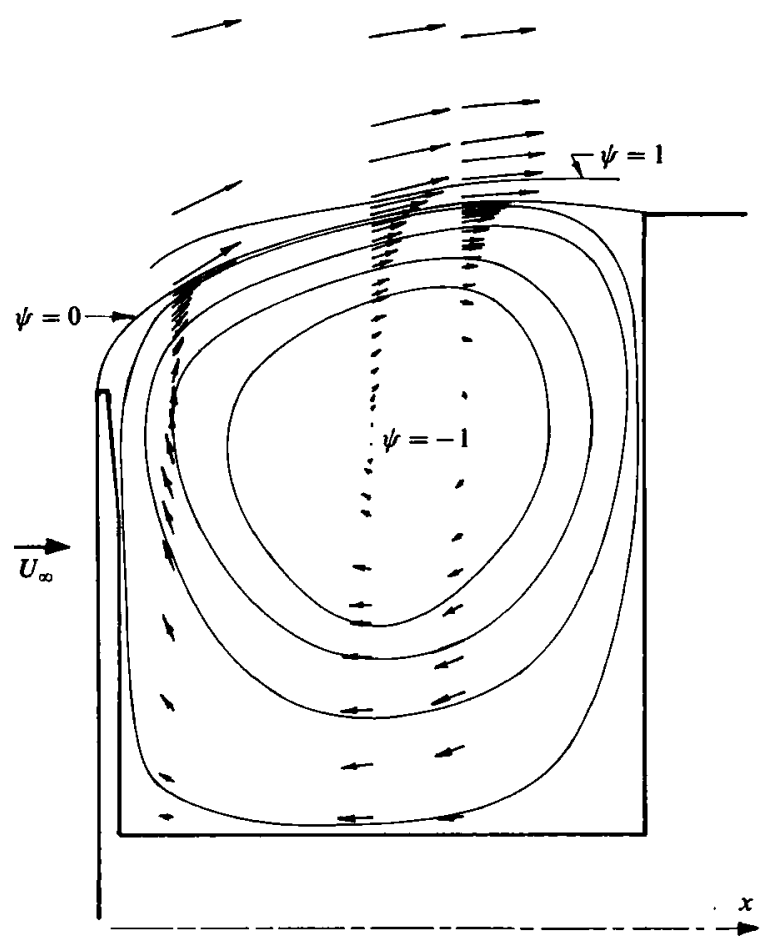

Figure 19. Velocity vectors and streamlines for the low-drag optimum geometry, $d_{1} / d_{2}=0.75, g^{*} / d_{2}=0.375$.

of $\bar{u}$ and $\bar{v}$ at three axial stations (cf. figure 17). The axisymmetric stream function $\psi$ defined by $u=(1 / r)(\partial \psi / \partial r), v=-(1 / r)(\partial \psi / \partial r)$ was calculated; some of the streamlines are freely drawn in figure 19. The streamline $\psi=-1$ is normalized to represent the total flow recirculating in the cavity; for comparison, the corresponding flux $\psi=1$ in the outer flow is also shown. At upstream infinity, this amount of flow is enclosed in a stream tube of diameter $0.343 d_{2}$. Figure 19 shows that the mean flow is dominated by a single vortical motion filling the cavity. The lower half of the cavity has roughly constant velocity in the upstream direction.

\section{Discussion of drag regimes and flow patterns}

In the preceding section we have identified and classified several flow regimes on the basis of drag minima in figure 5 and have, furthermore, argued that the flows in two of them (at the lower values of drag coefficient) can be modelled as cavity flows, 
with useful connections to free-streamline theory. In the following we review our measurements and observations and make some inferences about the phenomena occurring. Most striking is the comparison of regimes I and II, the low-drag and medium-drag regimes defined in figure 9.

In regime I the flows are evidently close to free-streamline flows (figures 7, 10, 12); the free shear layer reattaches onto or close to the shoulder of the rearbody; the velocity fluctuation level in the free shear layer is very low; correspondingly the Reynolds stress is lower, by a factor of about 3 , than the stress in plane, free turbulent shear layers; the low value corresponds to very low values of drag in this regime. One explanation for the depressed value is that the shear layer spanning a cavity is quite different from a free shear layer. This is what Gharib (1983) found at low Reynolds number, where so-called 'laminar' periodic oscillations are predominant and are modified by 'feedback' from the downstream edge. At high Reynolds number the so-called ' coherent' large structures of the turbulent layer could be similarly affected.

The curvature of the shear layer in the present geometries may also be a factor; a large reduction of Reynolds stress was observed by Castro \& Bradshaw (1976) in curved turbulent free shear layers.

In the medium-drag regime (II) the flow patterns at optimum drag also appear to be close to free-streamline flows (figures 7,10 ). However, the free shear layers are thicker and the level of velocity fluctuation and of Reynolds stress is much higher (figure 17) than in I. Although the layers are curved, Reynolds stresses are even higher than in a plane shear layer. In addition, a fairly high level of Reynolds stress exists deep in the cavity. This indicates that there is a large-scale oscillation involving the whole cavity; its coupling with the vortex structure in the free shear layer could account for the high Reynolds stress there. Such coupling could occur through the effect of the cavity oscillations on the reattachment region near the shoulder, which has upstream influence on the shear layer, etc. (Rockwell 1983). This large-scale cavity oscillation may be regarded as the beginning of a wake mode of the forebody (Gharib 1983), strongly influenced by the presence of the rearbody. In the twodimensional counterpart it may be expected that a splitter plate on the axis would stabilize the oscillations and extend regime I to larger gaps.

It is useful now to return to figure 2, where we had postulated what flow patterns might exist and in which region of the $(g, d)$-plane they might occur and to compare them with what actually occurs. Figure 2 shows steady, mean flows; to make the comparisons precisely it would be best to have mean-flow streamlines, especially the zero or dividing streamlines, which are the ones sketched in figure 2 and which determine the reattachment points. To obtain this, say from velocity measurements as was done for figure 19, is a task of large proportion that we were not able to undertake. Instead, we made the comparisons more qualitatively and subjectively by examining the flow pictures in figure 7, as well as others at our disposal, sorting them out as well as possible into the various types postulated in figure 2 and locating them on the $(g, d)$-plane. The position of the mean-zero streamline can then only be estimated, as somewhere near the middle of the visible free shear layer, and the position of reattachment guessed correspondingly. The result is shown in figure 20 , plotted on the same $g, d_{1}$ coordinates as figure 2. Although rather subjective and approximate, we believe that the classification is accurate enough to reveal several interesting results, as follows. (The reader is invited to check it with figure 7.) Most of the flow patterns postulated in figure 2 can be recognized in the flow pictures of figure 7 , but the regions of the $\left(g, d_{1}\right)$-plane in which they occur are rather different from those in figure 2 . Regimes $A, C$, and D are in approximately the same locations 


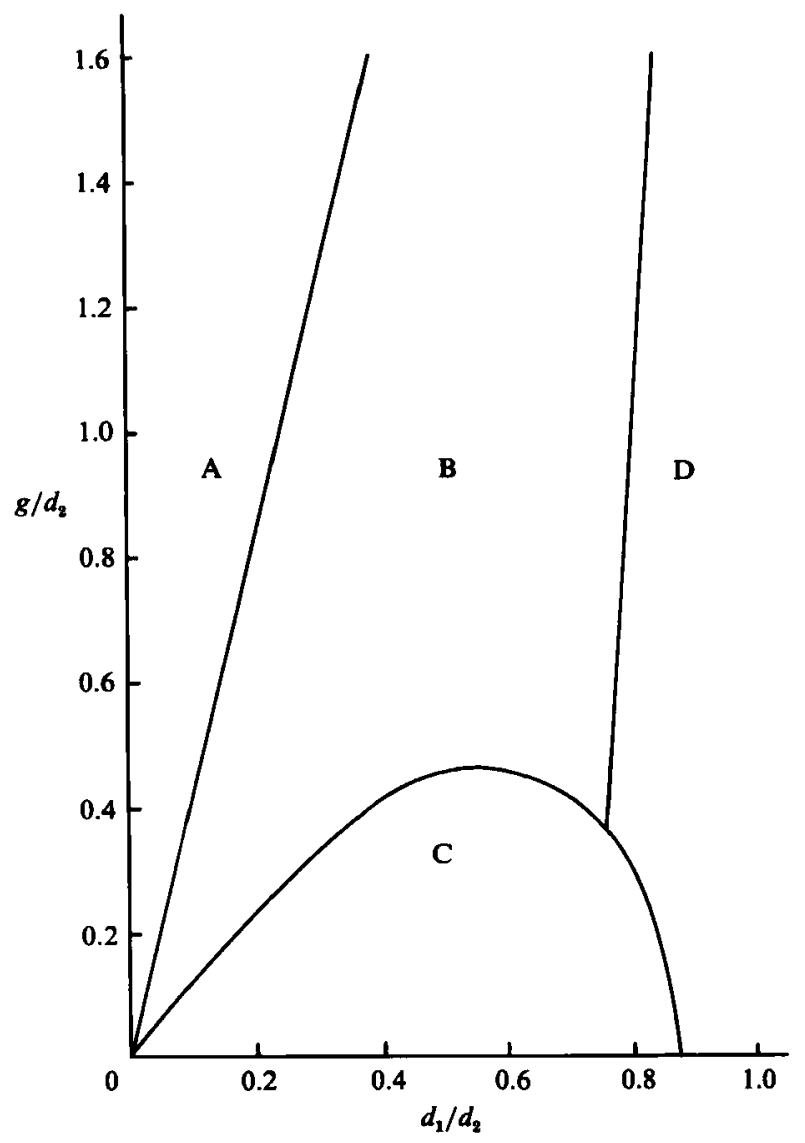

Figure 20. Flow regimes in the $(g, d)$-plane, inferred from flow pictures.

in the $(g, d)$-plane as in figure 2 , but their boundaries are considerably shifted, as expected.

We were not able to find any flows of type $A B$, in which the mean-wake-closure point is on the centre of the forebody. Admittedly it is highly unlikely to observe such a flow on an instantaneous picture, nevertheless we have the impression that the corresponding mean flow does not exist and that the boundary AB is one of discontinuous change. Determinations of the mean-flow fields would be valuable for settling this question and for more precisely defining figure 20 in general.

\section{Rounded corners on the axisymmetric rearbody}

The high drag on the rearbody alone could, of course, be drastically reduced, without assistance from a frontbody, by simply rounding its edges, or corners, sufficiently. A corner radius equal to one-eighth the body diameter is sufficient to reduce the drag of the rearbody face to nearly zero in the absence of any frontbody provided the Reynolds number is large enough that premature laminar separation does not occur (Polhamus 1957). For the rearbody used in the present experiments with an edge radius one-eighth the rearbody diameter $\left(r / d_{2}=0.125\right)$ and roughness on the face (sandpaper or a small disk) the drag coefficient was less than 0.01 for Reynolds numbers greater than 400000 based on the rearbody diameter. 

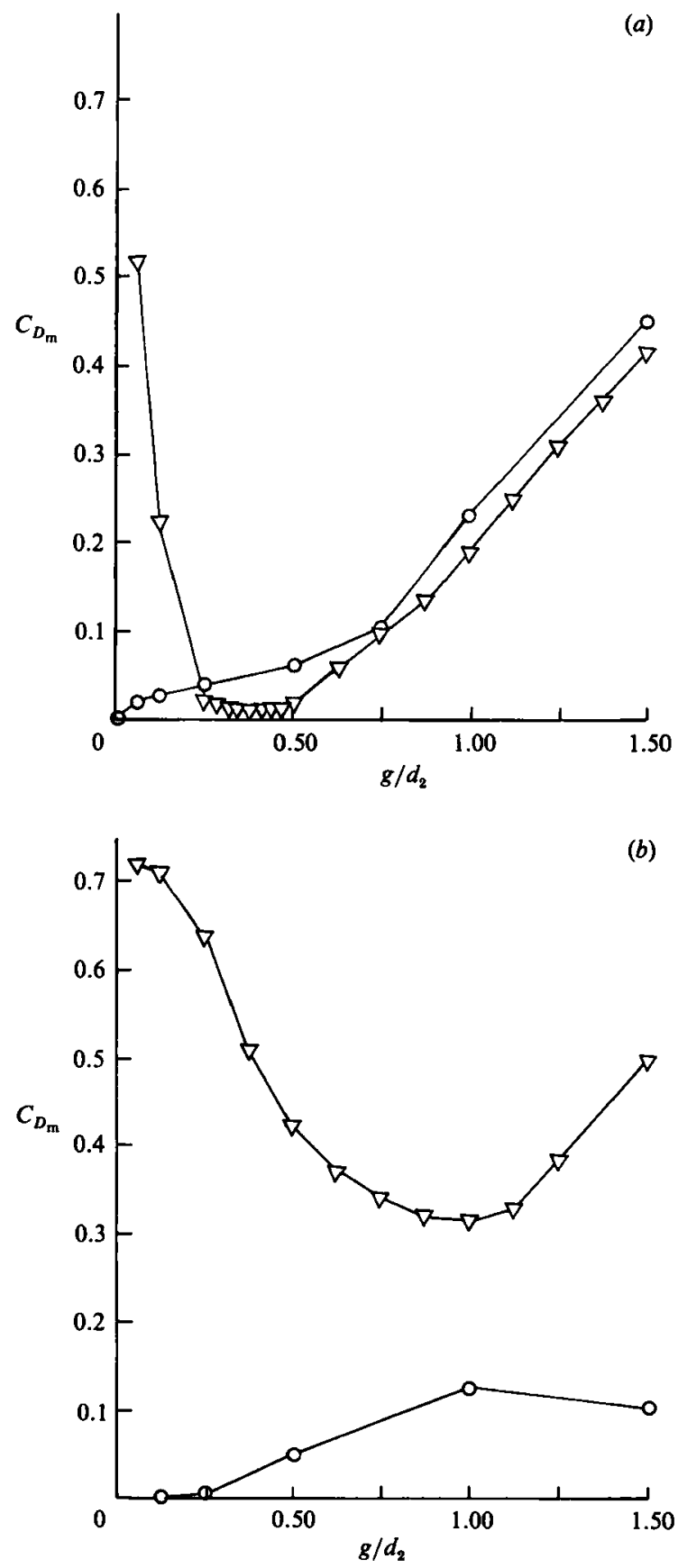

Figure 21. Effect of rearbody corner radius on forebody drag: $\nabla$, sharp corner; $\bigcirc$, round; $r / d_{2}=0.125$. (a) Axisymmetric cross-section, $d_{1} / d_{2}=0.75 ;(b) 0.25$.

It is of interest to see what effect a frontbody would have when the rearbody has rounded edges. Some results are shown in figure 21 for the axisymmetric model used in the previous experiments but with $r / d_{2}=0.125$. Comparison is made for two cases, $d_{1} / d_{2}=0.25$ and 0.75 , with and without rounded edges. The Reynolds number based on $d_{2}$ is 500000 . For $d_{1} / d_{2}=0.75$ (figure $21 a$ ) and small values of $g / d_{2}$ (less than 0.2 ) 
rounding of the rearbody results in a very large reduction of drag, because the flow which has reattached onto the rearbody face can develop suction on the rounded edges. For large gap ratios $\left(g / d_{2}>0.2\right)$, on the other hand, the rounding is not helpful. In fact, for intermediate gap ratios, where the square-edged body has very low drag, rounding of the edges is apparently detrimental. At these intermediate gaps the separation surface is reattaching very near the corner and the corner radius is likely to influence the reattachment process and the rearbody face pressure. For $d_{1} / d_{2}=0.25$ (figure $21 b$ ) rounding has a beneficial effect at all gaps, since reattachment is always on the rearbody face radially inboard of the corner and the flow is attached around the corner.

As the gap becomes large the rearbody becomes independent of the frontbody and in this situation rounding will be beneficial for all frontbodies. This can be seen in figure $21(b)$ for $g / d_{2}$ greater than 1.0 , where the drag coefficient begins to decrease. The results for the larger frontbody given in figure $21(a)$, however, do not extend to values of $g / d_{2}$ great enough to show the limiting behaviour. The asymptotic values of $C_{D}$ for the results in figure 21 are: for $d_{1} / d_{2}=0.25$,

$$
\begin{gathered}
C_{D} \rightarrow 0.72+1.15\left(\frac{1}{4}\right)^{2}=0.79, \quad \text { square corner, } \\
C_{D} \rightarrow 0+1.15\left(\frac{1}{4}\right)^{2}=0.07, \quad \text { rounded corner }
\end{gathered}
$$

and for $d_{1} / d_{2}=0.75$,

$$
\begin{gathered}
C_{D} \rightarrow 0.72+1.15\left(\frac{3}{4}\right)^{2}=1.37, \quad \text { square corner }, \\
C_{D} \rightarrow 0+1.15\left(\frac{3}{4}\right)^{2}=0.65, \quad \text { rounded corner. }
\end{gathered}
$$

A most interesting result of the rounding experiment is that for some gap ratios square edges are better than rounded ones. The reasons for this behaviour are not completely clear and a closer investigation of the reattaching flow for these conditions needs to be made.

\section{Square cross-section}

Many of the measurements made on the axisymmetric system were repeated for a system with square cross-section, i.e. a box with a square plate in front of it. The question naturally arises whether the same large reductions of drag can be realized as in the axisymmetric case. One might expect the situation to be less favourable since the separation surface leaving the front plate will not retain a square cross-section and so will not reattach smoothly everywhere onto the leading edges of the rearbody. A similar situation would exist in many practical applications. The following figures show the main results.

The variation of $C_{D}$ with $g / d_{2}$ is shown for two values of $d_{1} / d_{2}$ namely 0.25 and 0.75 (medium-drag and low-drag geometries respectively), in figure 22 along with the corresponding axisymmetric results. For the square configurations, $d_{1}$ and $d_{2}$ refer to lengths of the front-plate and rearbody sides respectively. The trends exhibited in figure 22 are roughly similar to those for the circular cross-section, although there are some important differences. For the low-drag frontbody $\left(d_{1} / d_{2}=0.75\right)$ the minimum value of $C_{D_{\mathrm{m}}}$ is considerably higher than for the circular section, about 0.07 compared to 0.01 , although it still represents a full order-of-magnitude decrease from the drag of the box alone, for which $C_{D_{\mathrm{m}}}=0.75$. In the axisymmetric case the separated flow conforms to the axisymmetric rearbody, but here there is a mismatch between the cross-sectional shape of the separation surface and the square section 


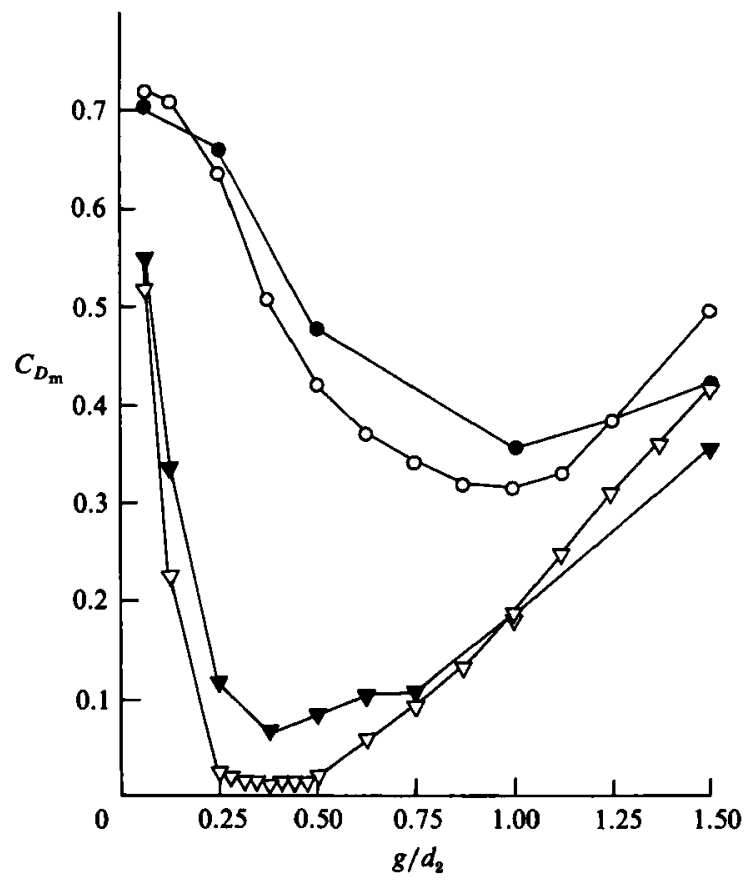

FIGURE 22. Effect of cross-section shape (sharp rearbody corner) on forebody drag: $O$, axisymmetric, $d_{1} / d_{2}=0.25 ; \nabla$, axisymmetric, $0.75 ; 0$, square, $0.25 ; \nabla$, square, 0.75 .

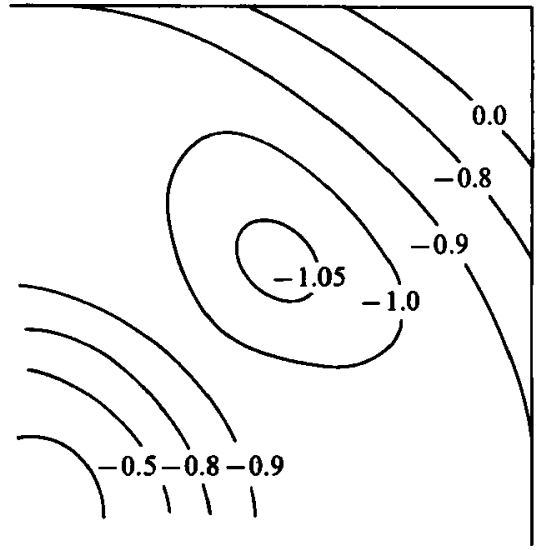

(a)

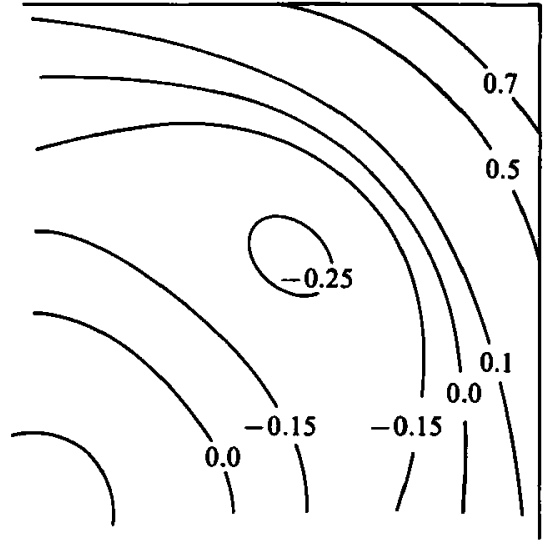

(b)

Fraure 23. Square-cross-section rearbody face pressure coefficient isolines. $(a) d_{1} / d_{2}=0.75$, $g / d_{2}=0.375, C_{D_{\mathrm{m}}}=0.07 ;(b) d_{1} / d_{2}=0.5, g / d_{2}=0.5, C_{D_{\mathrm{m}}}=0.22$.

of the rearbody onto which it is reattaching (a result observed in flow-visualization experiments but not adequately photographed) and this has a large effect on the minimum drag possible. For the medium-drag frontbody $\left(d_{1} / d_{2}=0.25\right)$ on the other hand, figure 22 shows rather little (percentage) difference between the two cases. Here the separation from the axisymmetric frontbody does not remain axisymmetric but forms an oscillating wake (see figure $7 a$ ) that does not smoothly reattach to the rearbody. Consequently the fact that the separation from the square frontbody does 
not remain square is not so important since there is a mismatch even for the axisymmetric case.

The significance of the mismatch of the separation surface with the square cross-section rearbody is more clearly seen in figure 23. Here pressure contours on one quadrant of the rearbody face are presented for a low-drag and a medium-drag optimum geometry (figures $23 a$ and $b$, respectively). Although most of the face of the low-drag geometry experiences pressures much less than free-stream static $\left(C_{p}=-1.0\right)$, the corner is exposed to pressure near stagnation $\left(C_{p_{\max }}=0.75\right)$. In comparison, the corresponding axisymmetric rearbody face (figure $8 c, g / d_{2}=0.375$ ) does not have any areas on which the pressure coefficient exceeds even -0.25 . In contrast, for the medium-drag geometries there is much less difference between the pressure distributions for the square cross-section (figure 23b) and the corresponding axisymmetric one (figure $8 a, g / d_{2}=0.75$ ). Failure of the separation surface to conform to the square rearbody is obviously of greater importance for low-drag geometries than for other configurations.

\section{Hemispherical frontbody}

There are many possible frontbodies besides thin disks; as a simple example that perhaps best contrasts with the disks we chose a hemisphere having the same diameter as the rearbody. With a hemispherical frontbody, at small gaps the forebody drag should be quite low since the situation is essentially that of a circular cylinder with a hemispherical nose. When the gap is larger the flow will be more like that over an axisymmetric cutout in a circular cylinder and the forebody system will experience the additional drag force due to this cutout. Results from a brief investigation of drag and pressure variations with changing gap for a hemisphere frontbody follow.

The drag coefficients for a hemispherical frontbody with $d_{1} / d_{2}=1.0$ are presented in figure 24 ; the coefficients here are uncorrected for blockage and skin friction. The continuous curve in figure 24 is for the disk $d_{1} / d_{2}=0.75$ while the dashed lines are coefficients predicted from (9) and will be discussed shortly. One is immediately struck by the negative values of $C_{D_{m}}$ for zero and small gap ratios, $C_{D_{m}}$ being -0.016 at zero gap. This negative drag is the consequence of blockage and should be corrected, using the results discussed in the Appendix of this paper. The net correction, $\Delta C_{D}=0.02 \pm 0.005$, indicates that the free-field drag coefficients are positive, though still extremely low.

For zero gap the body is a simple circular cylinder with a hemispherical nose. Corrected, the present results indicate that the forebody drag coefficient of this configuration is of the order of $C_{D_{c}}=0.005$. This result is somewhat lower than the few other values available in the literature. Hoerner (1965) integrated the (few) pressure measurements of Rouse \& McNown (1948) and obtained $C_{D}=0.01$ for a hemispherical forebody. Norris \& McGhee (1966) inferred $C_{D}=0.009$ by subtracting measured values of base drag and calculated values of skin-friction drag from the total drag measured on a circular cylinder with a hemisphere nose. A numerical calculation of the boundary-layer development on a hemisphere-cylinder (K. Kaups 1982, private communication) indicates that the skin-friction drag coefficient on the hemispherical nose is approximately 0.004 , which is consistent with the present results. Differences in experimental details and the difficulty in measuring small forces on a forebody make it difficult to assign an absolute value to $C_{D}$ for a hemispherical nose in a free stream. The value of $C_{D}$ is, however, certainly less than 0.01 and perhaps as low as $\mathbf{0 . 0 0 4}$. 


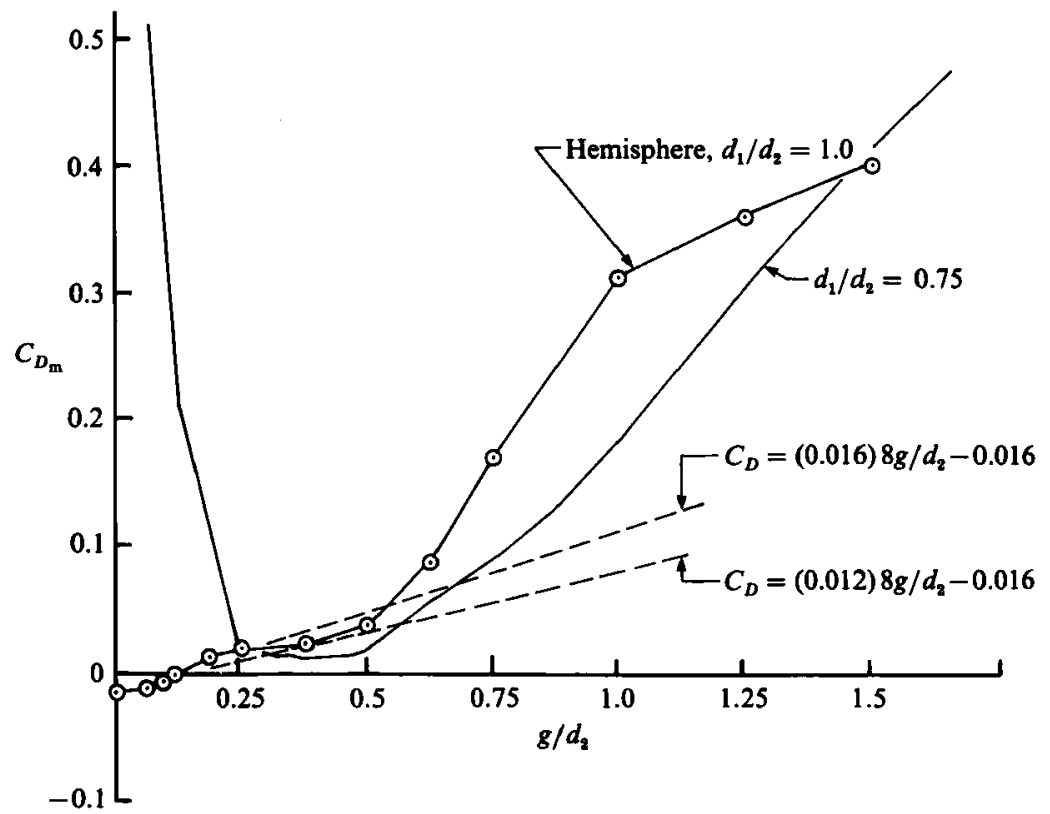

Figure 24. Measured drag coefficient for a hemispherical frontbody: $\odot$, hemisphere; - - , disk for comparison; ---, estimates of the forebody drag with a hemispherical frontbody based on (9).

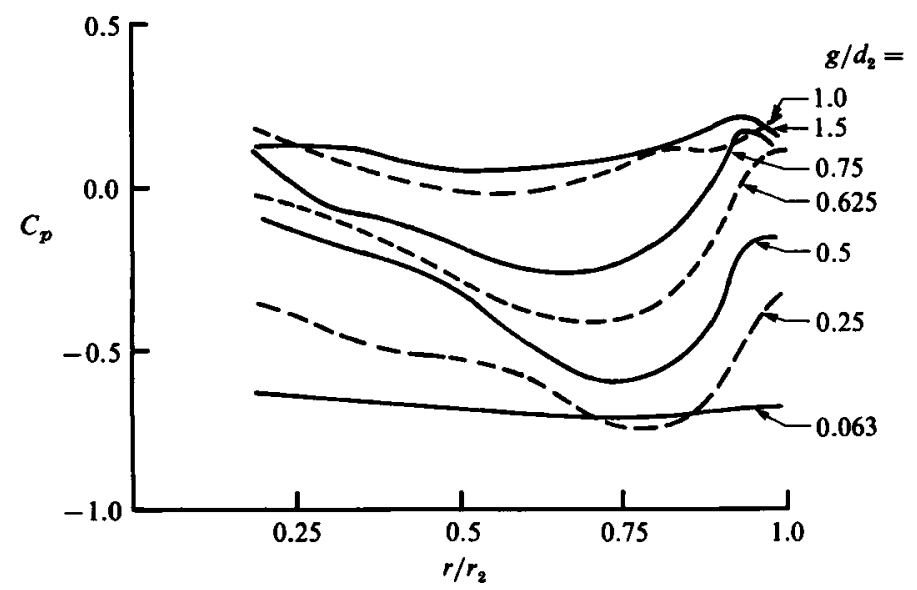

Figure 25. Rearbody-face pressure distributions with a hemispherical frontbody.

As the gap is increased from zero, figure 24 shows that the drag coefficient increases, rather slowly. The curves for the hemisphere and disk configurations cross near $g / d_{2}=0.25$ and beyond that value, until $g / d_{2}$ reaches 1.5 , the forebody system with disk has less drag than the one with the hemisphere. Over portions of this gap range the disk configurations with $d_{1} / d_{2}=0.625,0.688$, and 0.813 also have lower values of $C_{D}$ than the hemisphere case (see figure 5). An interesting feature of figure 24 is the discontinuity in the hemisphere drag-curve slope at $g / d_{2}=0.5$. Several of the very low-drag disk configurations, including the one shown here, also display an abrupt change in $C_{D}$ as the spacing is increased above a ratio of 0.5 . At asymptotically large gaps the isolated hemisphere, which has a free-field drag coefficient of about 0.42 
(Hoerner 1965), should be approximately equivalent to an isolated disk with $d_{1} / d_{2}=0.6$. From these force measurements then we do find the expected low forebody drag for the hemisphere configuration at small gaps. However, over a fairly wide range of larger values of $g / d_{2}$ the drag with hemispherical nose is larger than that which is possible with some disks.

The rearbody-face pressures for the hemispherical frontbody appear in figure 25 . A useful comparison to these distributions are those in figure $8(c)$, for the disk $d_{1} / d_{2}=0.75$. Qualitatively the distributions are similar but the levels of pressure are not. For example, at $g / d_{2}=0.5$ the minimum value of $C_{p}$ with the hemisphere is about -0.6 while with the disk the minimum value is approximately -0.9 . Again at $g / d_{2}=1.0$, the minimum value of $C_{p}$ is about 0 for the hemisphere and -0.6 for the disk.

Returning to figure 24 , two computed lines also appear in this plot. These lines are based on (9) which estimates the drag due to an axisymmetric cutout in a constant-diameter circular cylinder. The forebody drag is the sum of this cavity drag plus the friction drag on the upstream-facing surface of the frontbody. If we assume there is no change in the friction drag of the forward hemisphere face as it moves from zero to non-zero values of gap then the forebody drag coefficient is given by (9) plus the zero-gap value of $C_{D}$ which here is -0.016 uncorrected. That is

$$
C_{D}=C_{\tau} \frac{8 g}{d_{2}}-0.016 \text {. }
$$

Shear-stress measurements for the hemispherical frontbody were not obtained in this experiment and so the value of $C_{\tau}$ here is not precisely known. Since the shear layer in this case has negligible streamwise curvature it should be more like a plane mixing layer than are the shear layers downstream of disk frontbodies, so an appropriate value for $C_{\tau}$ might be close to that obtained in a plane mixing layer. Two values of $C_{\tau}$ have been chosen for this estimate, 0.012 inferred from the data of Liepmann \& Laufer (1947) and 0.016 from Wygnanski \& Fiedler (1970). With these two values for $C_{\tau}$, the dashed lines in figure 24 are obtained. The two estimates of $C_{D}$ bracket the measured values over the range from zero gap to $g / d_{2}=0.5$, at which point the measured drag curve changes slope. As previously noted, the curve for the diskforebody measurements also changes slope at this gap ratio, which is close to the value, 0.6 , shown in table 2 as the beginning of the medium-drag range. In measurements on a similar configuration but with ellipsoidal nose, Gharib noted a similar change of behaviour, which he called the beginning of the 'wake mode', at $g / d=0.65$.

This paper is based on work for the Ph.D. thesis by one of us (Koenig 1978). Some early results were presented at the 1976 General Motors Symposium on Aerodynamic Drag Mechanisms (Roshko \& Koenig 1978). The work was initiated with assistance from a Ford-Exxon Energy Research Grant and continued with support from National Science Foundation Grants ENG 75-03694 and ENG 77-23541. In addition, we are grateful for assistance in various forms from F. H. Clauser, D. E. Coles, P. E. Dimotakis, T. Liepmann and G. Lundgren.

\section{Appendix. Corrections for blockage and extraneous skin friction}

The effect of wind-tunnel wall constraint on forebody drag is to decrease the measured drag below the free-stream value. The increased velocity due to the wall constraint lowers the pressure on the forebody, whose surfaces are upstream facing. 
The change in drag coefficient

$$
\Delta C_{D_{1}}=\frac{-\alpha}{1-\alpha} \quad \text { where } \alpha=\frac{\text { a model }}{\text { a tunnel }}
$$

is independent of the forebody shape (Morel 1979; Koenig 1978). This result can be obtained from the well-known calculation of Prandtl \& Tietjens (1934) which shows that the downstream force in potential flow over a half body in a tunnel is

$$
C_{D_{\alpha}}=\frac{\alpha}{(1-\alpha)^{2}} \rightarrow 0 \text { for } \alpha \rightarrow 0,
$$

when the force includes the base pressure $C_{p_{\mathrm{b}}}$ in a slit across the body at downstream infinity, i.e.

Now

$$
\begin{gathered}
C_{D_{\alpha}}=C_{D_{\mathrm{f}}}-C_{p_{\mathrm{b}}} \\
C_{p_{\mathrm{b}}}=1-\left(\frac{U_{+\infty}}{U_{-\infty}}\right)^{2}=1-\left(\frac{1}{1-\alpha}\right)^{2}
\end{gathered}
$$

From (A 2) and (A 3)

$$
C_{D_{\mathrm{f}}}=-\frac{\alpha}{1-\alpha}=\Delta C_{D_{1}}
$$

For the wind tunnel and model on which our drag measurements were obtained, $\alpha=0.030$. Thus the measured values of $C_{D}$ should be increased by 0.031 to correct for wall constraint. The correction should be valid for the low-drag flows, for which there is no large separation that would change the effective (displacement) area of the rearbody. But it is only for the low values of drag that the correction is important. In fact, it is clear that the small values are of the same magnitude as the correction.

To estimate the skin-friction force on the metric segment of the rearbody is more problematic. Again for low-drag cases, the shear stress in the free shear layer impinging on the shoulder is of order $\tau=0.01 \rho U_{\infty}^{2}$. Although the reattached boundary layer might initially have a shear stress as high as this the stabilizing influence of the wall and the adverse pressure gradient should rapidly lower it, bringing it down to typical flat-plate values $\tau_{\mathrm{w}} \sim 0.0015 \rho U_{\infty}^{2}$ or less. For want of information about this interesting problem, we use the latter value, $C_{f}=0.003$, for an average value of skin-friction coefficient over the metric segment, which is 5 in. $(12.9 \mathrm{~cm})$ long compared to its $8 \mathrm{in} .(20.3 \mathrm{~cm})$ diameter. It gives a drag increase $\Delta C_{D_{2}}=0.01$. Thus the net correction to be added to the measured $C_{D}$, as defined in $(1)$, is $\Delta C_{D}=0.031-0.01=0.02 \pm 0.005$. Adding to this the uncertainty of 0.004 in the drag measurement itself (see §2, Experimental details), there is clearly considerable uncertainty as to the true values of the low-drag coefficients. It is for this reason that we deemed it important to present the data without the corrections, except in figure 9 and in figure 14 where comparisons are made with a model calculation.

In measurements of reattachment of a similar free shear layer downstream of a step, Bradshaw \& Wong (1972) found that the wall friction rapidly builds up to a value of $\tau_{\mathrm{w}}=0.003\left(\frac{1}{2} \rho U_{\infty}^{2}\right)$ then slowly decays. Thus $C_{f}=0.003$ appears to be a reasonable value for the average skin-friction coefficient.

While not so important, it is of interest to consider the blockage effects on a separated, high-drag configuration. A good example is the rearbody without the frontbody. As stated earlier, the value of the pressure drag on the front face, without correction for blockage or extraneous skin friction, is 0.72 . The side surfaces of the metric segment are now in separated, reverse flow, so the contribution of (reverse) 
friction force should be negligible. Applying the blockage correction of (A 1), i.e. $\Delta C_{D}=0.031$, then gives, for the corrected value, $C_{D_{\mathrm{c}}}=0.75$.

Another approach to the correction problem for this bluff body, using the well-known method of Maskell (1965) and making use of data for disks, was suggested to us by P. Bearman. For a disk (Morel 1980) the corrected, free-stream values of drag, base pressure and front-face average pressure respectively are

$$
C_{D \mathrm{c}}=1.12, \quad C_{p_{\mathrm{bc}}}=-0.36, \quad \bar{C}_{p_{\mathrm{fc}}}=0.76 .
$$

With blockage, Maskell's method predicts

$$
\frac{1-C_{p_{\mathrm{b}}}}{1-C_{p_{\mathrm{bc}}}}=\frac{C_{D}}{C_{D \mathrm{c}}}=1+\frac{C_{D}}{-C_{p_{\mathrm{bc}}}} \alpha .
$$

For $\alpha=0.030$, this implies $C_{D}=1.257, C_{p_{\mathrm{b}}}=-0.526$ and $\bar{C}_{p_{\mathrm{F}}}=0.73$. Thus the effect of blockage is to reduce the front-face force in the ratio $0.73 / 0.76$. Applying this to the face of the rearbody in our experiment gives for the corrected value $C_{D_{\mathrm{c}}}=(0.76 / 0.73) 0.72=0.75$, the same value as determined by (A 4).

For the configuration with hemispherical frontbody, the values of drag were again very low. The influence of blockage on this body should be the same as for the low-drag disks. The friction force requires knowledge of $C_{f}$ on the metric segment. Surprisingly the boundary-layer development just past the shoulder of a hemispherical forebody seems to be a subject missing in the literature. For estimation purposes we again take $C_{f}=0.003$ so that the net correction to the hemisphere at zero and small gaps is the same as for the low-drag disks.

\section{REFERENCES}

Bearman, P. W. 1971 An investigation of the forces on flat plates normal to a turbulent flow. J. Fluid Mech. 46, 177-198.

Bradshaw, P.\& Wong, F. Y. F. 1972 The reattachment and relaxation of a turbulent shear layer. J. Fluid Mech. 52, 113-136.

Brennen, C. 1969 A numerical solution of axisymmetric cavity flows. J. Fluid Mech. 37, 671-688.

CArmody, T. 1964 Establishment of the wake behind a disk. Trans. ASME D: J. Basic Engng 86, 869-882.

Castro, I. P. \& Bradshaw, P. 1976 The turbulence structure of a highly curved mixing layer. J. Fluid Mech. 73, 265-304.

Dimotakis, P. E. \& Lang, D. B. 1974 Single scattering particle laser-Doppler velocimetry. Bull. Am. Phys. Soc. No. 1145.

Dimotakis, P. E., LANG, D. B. \& Collins, D. J. 1978 Laser-Doppler velocity measurements in subsonic, transonic and supersonic boundary layers. In Proc. 3rd Intl Workshop in Laser Velocimetry.

Eifrel, G. 1913 The Resistance of the Air and Aviation, 2nd edn (translated by J. C. Hunsaker), pp. 54-60. London: Constable \& Co.

EPPLER, R. 1954 Contributions to the theory and applications of discontinuous flows. J. Rational Mechanics and Analysis 3, 591-644.

Garabedian, P. R. 1956 Calculation of axially symmetric cavities and jets. Pacific J. Mathematics 6, 611-684.

GhaRIB, M. 1983 The effect of flow oscillations on cavity drag, and a technique for their control. Ph.D. thesis, Aeronautics Department, California Institute of Technology.

Hoerner, S. F. 1965 Fluid Dynamic Drag, pp. 3-12. Hoerner Fluid Dynamics, Bricktown, New Jersey.

Joukowsky, N. E. 1930 Works of N. E. Joukowsky, vol. II, issue 3, Trans. CAHI, no. 41 (originally published in 1890). 
Kistler, A. L. \& TAN, F. C. 1967 Some properties of turbulent separated flows. Phys. Fluids Suppl. 10, S165-S173.

Kownia, K. 1978 Interference effects on the drag of bluff bodies in tandem. Ph.D. thesis, Aeronautics Department, California Institute of Technology.

LiEPMANN, H. W. \& LAUfER, J. 1947 Investigations of free turbulent mixing. NACA TN 1257.

LitTLe, B. H. \& WhIPKey, R. R. 1979 Locked vortex afterbodies. J. Aircraft 16, 296-302.

MaIR, W. A. 1965 The effect of a rear-mounted disc on the drag of a blunt-based body of revolution. Aero. Q. 16, 350-360.

Maskell, E. C. 1965 A theory of the blockage effects on bluff bodies and stalled wings in a closed wind tunnel. ARC Rep. \& Mem. 3400.

Morel, T. 1979 Theoretical lower limits of forebody drag. Aero. J. 83, 23-27.

Morel, T. \& BoHN, M. 1980 Flow over two circular disks in tandem. Trans. ASME I: J. Fluids Engng 102, 104-111.

Norris, J. D. \& McGheE, R. J. 1966 Effects of bluntness on the subsonic drag of an elliptical forebody. NASA TN D-3388.

OTA, T. 1975 An axisymmetric separated and reattached flow on a longitudinal blunt circular cylinder. Trans. ASME E: J. Appl. Mech. 42, 311-315.

Polнamus, E. C. 1957 Effect of nose shape on aerodynamic characteristics of a body of revolution having a fineness ratio of 10.94. NACA RM L.57F25.

Prandtl, L. \& Tretuens, O.G. 1934 Applied Hydro- and Aeromechanics (translated by J. P. Hartog), $\$$ 77-81, Dover.

Riabouchinsky, D. 1921 On steady fluid motion with free surfaces. Proc. Lond. Math. Soc. 19, 206-215.

Rockwell, D. 1983 Oscillations of impinging shear layers. AIAA J. 21, 645-664.

Roshko, A. 1954 A new hodograph for free streamline theory. NACA TN 3168.

Roshкo, A. 1955 Some measurements of flow in a rectangular cutout. NACA TN 3488.

Roshko, A. \& Koenia, K. 1978 Interaction effects on the drag of bluff bodies in tandem. In Aerodynamic Drag Mechanisms of Bluff Bodies and Road Vehicles (ed. G. Sovran, T. Morel \& W. T. Mason), pp. 253-286. Plenum.

Rouse, H. \& McNown, J. S. 1948 Cavitation and pressure distribution: head forms at zero angle of yaw. State University of Iowa Studies in Engineering. Bulletin 32.

SAUNDERS, W. S. 1966 Apparatus for reducing linear and lateral wind resistance in a tractor-trailer combination vehicle. U.S. Patent No. 3241876.

Stanbrook, A. 1966 Experimental pressure distributions on a plane-nosed cylinder at subsonic and transonic speeds. ARC Rep. d Mem. 3425.

STRÜCK, H. G. 1970 Discontinuous flows and free streamline solutions for axisymmetric bodies at zero and small angles of attack. NASA TN D 5634.

WARD, T. M. 1976 The hydrodynamics laboratory at the California Institute of Technology. Graduate Aeronautical Laboratory, California Institute of Technology.

Wygnanski, I. \& Fiedler, H. E. 1970 The two-dimensional mixing region. J. Fluid Mech. 41, 327.

ZdRavkovich, M. M. 1977 Review of flow interference between two circular cylinders in various arrangements. Trans. ASME I: J. Fluids Engng 99, 618-632. 